\title{
World Catalog of Bee Flies (Diptera: Bombyliidae): Corrigenda and Addenda
}

\author{
NEAL L. EVENHUIS ${ }^{1} \&$ DAVID J. GREATHEAD ${ }^{2}$ \\ 1 J. Linsley Gressitt Center for Research in Entomology, Bishop Museum, 1525 Bernice Street, Honolulu, \\ Hawai'i 96817-2704, USA; email: neale@ bishopmuseum.org \\ 2 NERC, Centre for Population Biology, Imperial College London, Silwood Park Campus, Ascot, Berkshire \\ SL5 7PY, UK; email: d.greathead@imperial.ac.uk
}

\section{Table of Contents}

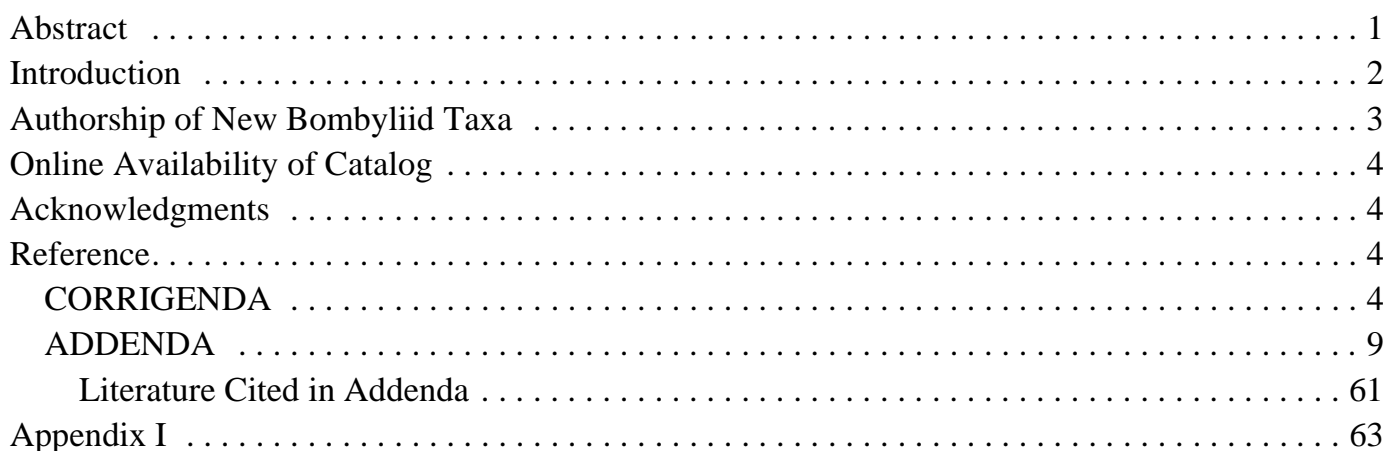

\begin{abstract}
A list of corrections and additions to the world catalog of bee flies, published in 1999 is presented. New taxonomic information presented in this paper includes the following 42 new species-group synonymies: Geron paramonovi Greathead (junior synonym of G. asiaticus Zaitzev), syn. n., Anastoechus olivaceus Paramonov, Anastoechus olivaceus var. corsikanus Paramonov, Anastoechus olivaceus var. corsicanus Zaitzev (all junior synonyms of A. nitidulus Fabricius), syn. n., Anastoechus leucochroicus Hesse (junior synonym of A. varipecten Bezzi, 1921), syn. n., Bombylius punctatus Fabricius, Bombylius sticticus Boisduval, Bombylius subluna Walker (all junior synonyms of Bombomyia vertebralis (Dufour)), syn. n., Bombylius flavescens Palm, Bombylius minor var. ochraceus Paramonov, Bombylius luteolus Evenhuis, Bombylius thapsinoides Evenhuis (all junior synonyms of B. minor Linnaeus), syn. n., Bombylius rhodius Loew (junior synonym of B. trichurus Pallas), syn. n., Bombylius aurulentus Wiedemann (junior synonym of Systoechus ctenopterus (Mikan)), syn. n., Bombylius lucidus Loew, Systoechus tesquorum Becker (all junior synonyms of Systoechus
\end{abstract}


gradatus (Wiedemann)), syn. n., Argyramoeba maculosa Sack, Anthrax ipiriensis Dils \& Weyer, Anthrax tajikistanus Evenhuis \& Greathead (all junior synonyms of Anthrax chionostigma Tsacas), syn. n., Spogostylum nigrum Austen (junior synonym of Anthrax lucidus (Becker)), syn. n., Argyromoeba velox Loew, Anthrax hellenicus François (all junior synonyms of Anthrax virgo Egger), syn. n., Exoprosopa dispar var. interrupta Paramonov, Exoprosopa dispar ssp. paramonovi Evenhuis (all junior synonyms of E. dispar Loew), syn. n., Thyridanthrax albicingulus Austen, Thyridanthrax amoenus Austen (all junior synonyms of Exhyalanthrax melanchlaenus (Loew)), syn. n., Thyridanthrax innocens Austen (junior synonym of Exhyalanthrax muscarius (Pallas)), syn. n., Anthrax venusta Meigen, Anthrax margaritifer Dufour, Anthrax dolosa Jaennicke, Anthrax turbidus Loew (all junior synonyms of Villa fasciata Meigen), syn. n., Villa orientalis Zaitzev (junior synonym of Villa fasciculata Becker), syn. n., Anthrax humilis Ruthe, Anthrax mucida Zeller, Anthrax pygarga Loew, Anthrax albulus Loew, Villa quinta Becker (all junior synonyms of Villa ixion (Fabricius)), syn. n., Anthrax faustina Osten Sacken, Anthrax alternata var. fulvipes Coquillett, Villa lateralis ssp. semifulvipes Painter in Painter \& Painter (all junior synonyms of Villa lateralis (Say)), syn. n., Petrorossia chraminensis Zaitzev, Petrorossia dobrogica Dusa (all junior synonyms of Petrorossia hespera (Rossi)), syn. n.; the following new synonymy in genus-group names: Gazlia Zaitzev (junior synonym of Xeramoeba Hesse), syn. n.; and the following 11 new combinations: Apolysis barri (Tabet) (from Oligodranes), comb. n., Apolysis nauseosus(Tabet) (from Oligodranes), comb. n., Parageron loewi (Becker) (from Usia), comb. n., Chalcochiton carmelitensis (Becker) (from Cytherea), comb. n., Exoprosopa rivularis Meigen (from Heteralonia), comb. n. Micomitra shelkovnikovi (Paramonov) (from Pterobates), comb. n., Xeramoeba albula (Zaitzev) (from Gazlia), comb. n., Xeramoeba kinereti (Zaitzev) (from Chiasmella), comb. n., Xeramoeba stackelbergi (Zaitzev) (from Chiasmella), comb. n., Xeramoeba turkmenica (Zaitzev) (from Chiasmella), comb. n. Two species are returned to their original genus: Exoprosopa dispar Loew (returned to Exoprosopa from Heteralonia), comb. rev., and Spogostylum nitidum Austen (returned to Spogostylum from Anthrax), comb. rev. Three species in Pipunculopsis were invalid when originally proposed by Zaitzev in 2001. They are hereby validated with the criteria fulfilling Article 16.4.2 and the original authorship of Zaitzev should be kept since he provided the information to validate the names herein.

Key words: Bombyliidae, catalog, taxonomy, distribution

\section{Introduction}

Since publication of the world catalog of Bombyliidae (Evenhuis \& Greathead, 1999; publication date: 10 November 1999) a number of corrections have been found by us. Some additional corrections or expanded information has also been made available to us by others, for which we are grateful. We here provide a list of corrections including 6 speciesgroup names inadvertently omitted from the original catalog and a list of addenda that includes 85 new species-group names, 2 new genus-group names of Bombyliidae, and numerous new distributional and corrected data published since our catalog. Including the number of new synonymies since publication of our catalog plus the 85 new species-group taxa, there are a total of 4,583 species of Bombyliidae now known. 
Exoprosopa still retains honors as the most speciose genus (338 species), followed by Bombylius (300), Anthrax (267), and Villa (257). Other genera with more than one hundred species include Geron (179), Systropus (155), Heteralonia (138), Apolysis (119), Systoechus (118), and Ligyra (109).

\section{Authorship of New Bombyliid Taxa}

Table 1 gives statistics on the numbers of new taxa described by various authors of Bombyliidae. Only those authors who have authored or co-authored over 100 new speciesgroup names are listed. The columns include total numbers of new genera and total numbers of new species as well as numbers of taxonomically valid taxa for genera and species per author and percent validity.

TABLE 1. Authors who have described more than 100 species-group names of Bombyliidae.

\begin{tabular}{lllllll}
\hline author & genera & $\begin{array}{l}\text { valid } \\
\text { genera }\end{array}$ & $\begin{array}{l}\text { \% } \\
\text { valid }\end{array}$ & species & $\begin{array}{l}\text { valid } \\
\text { species }\end{array}$ & $\begin{array}{l}\text { \% } \\
\text { valid }\end{array}$ \\
\hline Hesse & 18 & 13 & 0.72 & 674 & 580 & 0.86 \\
Bezzi & 32 & 21 & 0.70 & 431 & 350 & 0.81 \\
Paramonov & 12 & 6 & 0.50 & 411 & 313 & 0.76 \\
Loew & 24 & 16 & 0.67 & 316 & 244 & 0.77 \\
Evenhuis & 29 & 23 & 0.79 & 265 & 230 & 0.87 \\
Hall & 7 & 6 & 0.86 & 240 & 222 & 0.93 \\
Macquart & 17 & 13 & 0.76 & 286 & 171 & 0.60 \\
Zaitzev & 3 & 1 & 0.33 & 178 & 169 & 0.95 \\
Greathead & 11 & 10 & 0.91 & 161 & 152 & 0.94 \\
Walker & 4 & 2 & 0.50 & 221 & 148 & 0.67 \\
Wiedemann & 8 & 5 & 0.62 & 181 & 139 & 0.77 \\
Coquillett & 9 & 7 & 0.78 & 145 & 123 & 0.85 \\
Yeates & 1 & 1 & 1.00 & 113 & 112 & 0.99 \\
Bowden & 8 & 7 & 0.88 & 119 & 112 & 0.94 \\
Becker & 16 & 8 & 0.50 & 167 & 101 & 0.60 \\
Painter & 5 & 5 & 1.00 & 113 & 98 & 0.87 \\
\hline & & & & & & \\
\hline
\end{tabular}


The world catalog of Bombyliidae is available now on the web at: [http://hbs.bishopmuseum.org/bombcat/] where the original version and current versions are available as pdf files.

\section{Acknowledgments}

We are grateful to the following who have given us corrections for errors in the original catalog and who have assisted in augmenting data on types, distributions, collectors, or dates of publication: Adrian C. Pont (Oxford), Carlos Lamas (Rio de Janeiro), Christine Lambkin (Canberra), Valery Korneyev (Kiev), Jozef Dils (Stabroek), Patrick Grootaert (Brussels), Marcos Báez (Tenerife), V.F. Zaitzev and Izya Kerzhner (St. Petersburg).

\section{Reference}

Evenhuis, N.L. \& Greathead, D.J. (1999) World Catalog of Bee Flies (Diptera: Bombyliidae). Backhuys Publishers, Leiden. xlviii +756 pp.

\section{CORRIGENDA}

\section{page(s) item}

xiii line 24 up — "Gerontinae" should read "Gerontini".

xiv line 7 up - "Eclimini" should read "Ecliminae" and not be indented.

xvi line 19 up - Heteralonia row: move "32" from AU to OR

xvi line 31 up - Aphoebantus row: change "64" to "65" under NE column.

xxiii line 25 up - "HLDH" should read "HLMD".

16 lines 12-13 up - Usia anus Macquart ; delete "(= male S of Usia vicina Macquart)".

18 line 8 down - Usia cuprea Macquart: "10S in UMO*; $1 \mathrm{~S}$ in MNHN*" should read "H in MNHN".

18 line 10 down - Usia gagathea Macquart: "1S" should read "H".

19 line 6 up - Usia vestita; delete "4S in UMO*".

48 line 20 down - Bombylius insularis Bigot: "in BMNH*" should read "in MNHN*"

53 lines 5 and 6 down - "testacea" should read "testea"

107 line 19 down - Bombylius unicolor Bigot: "Italy" should read "Italy (Sicily)".

107 line 19 up — "(Latreille, 1810: 414)" should read "(Latreille, 1810: 443)"

123 line 9 down - Bombylius medius var. pallipes; delete " $2 \mathrm{~S}$ in UMO*". 
144 line 18 up - "Palaearctic" should read "Afrotropical".

161 lines 19, 23, and 26 up - "Painter, 1933a" should read "Painter, 1933b".

174-185 for the following species (in order of presentation in the catalog), the original genus "Systeochus" should read "Systoechus":

aurifacies ssp. maliensis
badipennis
bombycinus var. bedfordi
candidulus
canicapillus
cellularis
rufiarticulatus
damarensis
exiguus
flavicapillus
fumipennis
glabellula
leucophaeus var. gallicus
heteropogon
kalaharicus
leucostictus
longirostris
melampogon
monticolanus
neglectus
nigripes var. plebeius
niveicomatus
phaeopterus
pumilio
robustus
scabrirostris
silvaticus var. turneri
eupogonatus
srilankae
submicans
titan
ventricosus
waltoni

austeni
badius
bombycinus var. pallidispinis
candidus
canipectus
chlamydicterus
croeceipilus
deceptus
exilipes
flavospinosus
fumitinctus
goliath
gradatus var. validus
horridus
lacus
lightfooti
unicolor
mentiens
montuosus
nigribarbus var. falsus
nigripes var. nomteleensis
oreas
polioleucus
quasiminimus
rudebecki
segetus
simplex
somali
stevensoni
subulinus
transvaalensis
vulgaris
xanthoplocamus

autumnalis albibarbis
bechuanus
brunnibasis
canescens
castanealis
chrystallinus
sulphureus ssp. orientalis
eremophilus
faustus
fuligineus
fusciventris
gomezmenori
grandis
inordinatus
leoninus
litoralis
marshalli
montanus
namaquensis
nigripes
nivalis
pallidipilosus
poweri
rhodesianus
salticolus
silvaticus
sinaiticus
spinithorax
subcontiguus
tesquorum
tumidifrons
vulpinus
xerophilus

178 line 8 up - "Portugal" should read "Morocco".

188 line 1 down - Chad should be moved to under Afrotropical on p. 187.

193 line 26 up - Conophorus melanoceratus Bigot: "in MCZ*" should read "in $\mathrm{BMNH}^{*}$.

197 line 2 down - Legnotomyia fascipennis Bezzi: "[L here designated (see Appendix 1) in ZMHB*]" should read "[1S in ZMHB*]"

231 line 3 down - Amictus pictus; delete "1S in UMO*".

231 line 8 up - Amictus validus; delete " $5 \mathrm{~S}$ in UMO*".

232 lines 19-20 up - Callistoma fascipennis: " $1 \mathrm{~S}$ in UMO*" should read "T in 
UNKN".

291 line 12 up - Musca anthrax Schrank: "here designated (see ..." should read

"here designated by Evenhuis \& Thompson (see ...".

313 line 6 down - Anthrax plurinota: " $H$ " should read " $L$ designated by Marston (1970: 111)".

343 line 16 down: "Palaearctic: Eritrea, Saudi Arabia" should read "Afrotropical: Eritrea. Palaearctic: Saudi Arabia.

345 line 5 up - Pteraulax braunsi Bezzi: "MNSM" should read "MSNM".

347 lines 5-6 up - move Corycetta genus-group entry to p. 394 under Heteralonia (Zygodipla).

347 between lines 6 and 7 up - move Cladodisca genus-group entry to here from p. 383 and change to read as follows:

"CLADODISCA Bezzi, 1922b: 105 (as subgenus of Exoprosopa Macquart).

Type species: Exoprosopa munda Loew, 1869, by monotypy."

[Note: all subsequent descriptions and type designations by Bezzi are nomenclaturally irrelevant.]

line 16 up - Exoprosopa baccha Loew: add "1S in UMO" after "14S in ZMHB*".

line 7 down - Exoprosopa minois Loew: "[= Greece, Syria, or Turkey]" should read "[= Turkey]". Add "; $1 \mathrm{~S}$ in UMO* after $12 \mathrm{~S}$ in ZMHB*" [which was inadvertently placed under minos Meigen in the Catalog].

line 14-15 down - Anthrax minos Meigen; delete "1S in UMO*.

383 lines 8-10 up - move Cladodisca genus-group entry to p. 347.

390 line 1 up - "Afrotropical" should read "Palaearctic".

393 line 20 down -Exoprosopa delineata Becker: "[H in ZMHB]" should read "[H in ZMHB*]".

394 between lines 18 and 19 up - move Corycetta genus-group entry on p. 347 to here.

405 line 22 up - Hyperalonia flavosparsa Bigot: "[H in BMNH*]" should read "[L designated by Painter \& Painter (1974: 146) in BMNH*]".

422 line 8 up - Stomylomyia leonina Bigot: "[2S in UMO*]" should read "[1S in UMO*'".

422 line 10 up -Tomomyza europaea Loew: "[= Greece, Syria, or Turkey]” should read "[= Turkey]".

429 line 21 down - Anthrax unicinctus Bigot - " $H$ " should read "L designated by Painter \& Painter (1974: 276)".

434 line 21 down - Anthrax tangerinus Bigot - "S" should read " $\mathrm{H}$ ".

444 line 3 down — "1928b" should read "1928a".

480 line 8 down - Anthrax bifenestratus Bigot - "S" should read "L designated by Painter \& Painter (1962: 87)".

480 line 12 up - Anthrax perspicillaris Loew: add "1S in UMO" after "19S in 
ZMHB*".

487 line 7 down - Anthrax albulus Loew: "3S in UMO*" should read "1S in UMO*".

495 line 4 down - Anthrax stoechades Jaennicke: "[1S in UMO*]" should read "[T (lost) in SMFD]".

$513 \quad$ line 23 up: "1S in UMO*" should be deleted.

540 line 20 down - "'collection in ZMKU" should read "collection in SIZK".

550 between lines 3 and 4 up, add the following reference under Báez, M.:

1991 El género Thiridanthrax en las Islas Canarias (Diptera, Bombyliidae). Bol. Asoc. Esp. Entomol. 15: 53-56. [31 December 1991+]

601 line 11 up — "Pal, T.K." should read "Pal, Tharun Kumar (1953- )".

618 line 14 up — "Tucker, Elbert S." should read "Tucker, Elbert Stephen".

618 between lines 11 and 12 up - add the following author and reference:

TURTON, WILLIAM (1762-1835)

1801 A general system of nature, through the three grand kingdoms, of animals, vegetables, and minerals: systematically divided into their several classes, orders, genera, species, and varieties, with their habitations, manners, economy, structure and peculiarities. Translated from Gmelin's last edition of the celebrated Systema Naturae, by Sir Charles Linné: amended and enlarged by the improvements and discoveries of later naturalists and societies, with appropriate copper-plates. Vol. III. Lackington, London. 784 p.

628 change dates for Zaitzev articles in Entomol. Obozr. as follows (information given below is original year, new year, changed publication date, and changed annotation):

"1989b" should read "[1990]" “[2 February 1990]”

"[Date of receipt at ZIS; ... ]"

"1991b" should read "[1992]" "[9 March 1992]"

"[Date of receipt at ZIS; ... ]"

"1995" should read "[1996]" "[8 July 1996]"

"[Date of receipt at ZIS; ... ]"

"1996b" should read "[1997]" "[30 February 1997]”

"[Date of receipt at ZIS; ... ]"

"1997" should read "[1998]" "[30 January 1998]"

"[Date of receipt at ZIS; ... ]"

682 line 4 up — "G. olivieri" should read "G. olivierii".

683 line 19 down - "hair ocellar triangle" should read "hair on ocellar triangle"

684 delete first line down (repeats from previous page).

684 line 10 down - "(Cadeau de Kerville)" should read "(Gadeau de Kerville)". 
zоOTAXA 684 line 14 down - "(Tereul, ZMAN)" should read "(Tereul, KBIN)".

300688 line 30 down — add "BMNH" to depositories for Bowker.

688 line 4 up — augment vital date for Calaby: "(1922-1998)"

689 line 10 down - augment name and vital dates: "Charykuliev, Dzhuma Muradovich (1931- )".

690 line 17 down — add "ZMHB" to depositories.

690 line 19 down - augment name: "Doria, Laura ( - [D])".

691 line 1 up — add "Spain" to countries for Guichard.

691 line 26 up - augment name: "Gillon, Yves".

691 line 27 up — augment name: "Ghorpade, Kumar Dattajaro".

691 line 13 down — augment name and vital dates: "Forbes, Gregory Scott (1947)".

693 line 9 up — add vital dates "(1947_ _ )" for Lachaise.

695 line 13 down — add vital dates "(1963- )" for Miksch, Gabriel.

695 line 15 down - augment name and vital dates: "Mittre, Marius-Henri-Casimir (fl. 1831-1848)".

696 line 30 up — "Peña, Luis E" should read "Peña [Guzmán], Luis E".

697 line 17 down — add comma between "Riek" and "Edgar".

699 line 3 up - augment name and vital dates: "Van Someren, Gurber (19131997)".

699 line 15 up - augment name: "Tucker, Elbert Stephen".

700 line 2 down — augment name and vital dates: "Vaucher, Hugues (1922- )".

$700 \quad$ line 14 down - augment name and vital dates: "Wagner, Émil Roger (1868[D])".

$700 \quad$ line 13 up — augment vital dates for Wu Hong: "(1960- )".

701 line 11 down - delete "Ing."

734 line 19 down - mutilus Loew: change page from "437" to "479".

Additions to Collectors of Types (p. 687-701):

Bellier de la Chavignerie, Émile (1821-1871) Corsica, Sicily

Escalera, Manuel Martinez de la (fl. 1907-1914) Spain

MNMS

Germain (fl. 1857)

Chile

$\mathrm{BMNH}$

Lucas, J A W

Spain

KBIN

Mayet, Valéry (fl. 1879-1903)

Tunisia

KBIN

Seriziat, Charles-Victor-Émile (fl. 1868-86)

Algeria

UMO

\section{Taxa Omitted:}

14 add the following below incisus in the genus Usia: 
kassemi

Oligodranes kassemi Paramonov, 1947: 220. Nomen nudum.

70 between lines 19 and 20 down: add the following junior synonym of Toxophora amphitea:

Toxophora fucata Osten Sacken, 1877: 267. Nomen nudum.

321 between lines 4 and 5 up: add the following as a junior synonym of Satyramoeba hetrusca:

Bombylius haeruseus: Turton, 1801: 684 (incorrect subsequent spelling of Anthrax hetrusca Fabricius, 1794).

340 add the following between persicus and pusillus in the genus Aphoebantus:

petiolata

Aphoebantus petiolata Hull, 1973: 322. TYPE LOCALITY: "Southwestern United States" [T in UNKN].

DISTRIBUTION: Nearctic: "Southwestern United States".

411 add the following as a junior synonym under Anthrax tantalus:

Hyperalonia hyx Brunetti, 1909b: 439. Nomen nudum.

478 add the following between indigenus and kozlovi in the genus Thyridanthrax:

insularis

Thyridanthrax insularis Báez, 1991: 54. TyPE LOCALITY: Canary Islands [H in MABC].

DISTRIBUTION: Palaearctic: Canary Is.

\section{ADDENDA}

The following are additions to the catalog based on papers published since the catalog went to press and new information from us based on recent material examined. For new species added to the catalog, full entries are given below following the same format as the original catalog. For additions of data to previously described species (new synonymies, distributions, etc.), only the original combination, author and date are given under the species heading. Format of each species listing follows that of the original catalog including collection abbreviations. Depositories additional to those listed in the original catalog are identified below. Types seen by at least one of us are marked with an asterisk.

N.B.: Since publication of our catalog, South Africa (Northern Province) has changed its name to South Africa (Limpopo) 
Add to list of type depository abbreviations:

300 FSCA Florida State Collection of Arthropods, Gainesville, Florida, USA

NMNW National Museum of Namibia, Windhoek Namibia.

NSMT National Science Museum, Tokyo, Japan.

TAU Department of Zoology, Tel Aviv University, Tel Aviv, Israel

\author{
Subfamily USIINAE Becker \\ Tribe APOLYSINI Evenhuis \\ Genus APOLYSIS Loew
}

\title{
arenicola
}

Apolysis arenicola Tabet, 1979. Nomen nudum.

Apolysis arenicola Tabet, 2001d: 53. TYPE LOCALITY: USA (Idaho) [H in CAS].

DISTRIBUTION: Nearctic: USA (Idaho).

\section{barri}

Oligodranes barri Tabet, 1979. Nomen nudum.

Oligodranes barri Tabet, 2001d: 54. TYPE LOCALITY: USA (Idaho) [H in CAS].

COMB. N.

DISTRIBUTION: Nearctic: USA (Idaho).

\section{brachycera}

Apolysis brachycera Hesse, 1938.

DisTRIBUTION: add: Afrotropical: Namibia.

\section{brevirostris}

Apolysis brevirostris Hesse, 1975.

DistRIBUTION: add: Afrotropical: Namibia.

\section{eremophila}

Apolysis eremophila Loew, 1873.

Distribution: add: Palaearctic: Croatia.

\section{flavifemoris}

Oligodranes flavifemoris Hesse, 1975.

DisTRIBUTION: add: Afrotropical: Namibia.

\section{humilis}

Apolysis humilis Loew, 1860.

DISTRIBUTION: add: Afrotropical: Namibia. 
maherniaphila

Apolysis maherniaphila Hesse, 1938.

DISTRIBUTION: add: Afrotropical: Namibia.

namaensis

Oligodranes namaensis Hesse, 1938.

DISTRIBUTION: add: Afrotropical: Namibia.

nauseosa

Oligodranes nauseosus Tabet, 1979. Nomen nudum.

Oligodranes nauseosus Tabet, 2001d: 55. TyPE LOCALITY: USA (Idaho) [H in CAS].

COMB. N.

DISTRIBUTION: Nearctic: USA (Idaho).

\section{thornei}

Apolysis thornei Hesse, 1938.

DISTRIBUTION: add: Afrotropical: Namibia.

Tribe USIINI Becker

Genus PARAGERON Paramonov

gratus

Usia grata Loew in Rosenhauer, 1856.

Distribution: add: Palaearctic: Jordan; delete: Palaearctic: Canary Islands.

loewi

Usia loewi Becker, 1906. COMB. N. (transferred to Parageron from Usia).

\section{Genus USIA Latreille}

VOLUCCELLA Fabricius, 1794: 412. Type species: Voluccella florea Fabricius, 1794, automatic (by designation of type species for Usia Latreille).

USIA Latreille, 1802: 430 (unjustified new replacement name for Voluccella Fabricius, 1794). Type species: Voluccella florea Fabricius, 1794, by subsequent designation of Latreille (1810: 443).

Note: The remark under Usia in the original catalog was written before the new I.C.Z.N. Code was published. An application to the I.C.Z.N. is no longer necessary. The following statement can resolve the situation. 
[By virtue of I.C.Z.N. Code Article 23.9.1, whereby the criteria of both Articles 23.9.1.1 and 23.9.1.2 are met, Usia Latreille, 1802 is hereby valid and a nomen protectum by action of Article 23.9.2. Voluccella Fabricius, 1794, is hereby a nomen oblitum. A list of works giving evidence of the validity of Usia by use of its name as a valid taxon is given in the Appendix to this work.]

Subgenus MICRUSIA Evenhuis

engeli

Usia engeli Paramonov, 1950 (new move to subgenus Micrusia from nominate subgenus).

sicula

Usia sicula Egger, 1859.

DistRIBUTION: add: Palaearctic: Greece (Rhodes).

versicolor

Bombylius versicolor Fabricius, 1787.

DisTRIBUTION: add: Palaearctic: Italy (Sicily).

Subgenus USIA Latreille

aenea

Bombylius aeneus Rossi, 1794.

Distribution: delete: Palaearctic: Czech Republic, Slovakia, Switzerland.

atrata

Voluccella atrata Fabricius, 1798.

Distribution: add: Palaearctic: France (Corsica); delete: Palaearctic: Hungary, Slovakia, Switzerland.

bicolor

Usia bicolor Macquart, 1855.

DistRIBUTION: add: Palaearctic: Greece (Lesbos), Italy (Sicily), Jordan.

ignorata

Usia ignorata Engel, 1932.

DISTRIBUTION: add: Palaearctic: Jordan.

pallescens

Usia pallescens Becker, 1906.

DISTRIBUTION: add: Palaearctic: France, Portugal. 
similis

Usia similis Paramonov, 1950.

DISTRIBUTION: add: Palaearctic: Malta.

\section{Subfamily PHTHIRIINAE Becker \\ Tribe PHTHIRIINI Becker \\ Genus PHTHIRIA Meigen}

canescens

Phthiria canescens Loew, 1846.

Distribution: delete: Palaearctic: Finland, Slovakia, Switzerland.

conspicua

Phthiria conspicua Loew, 1846.

DisTRIBUTION: add: Palaearctic: Greece (Crete), Italy (Sicily), Spain (Mallorca).

\section{freidbergi}

Phthiria freidbergi Zaitzev, 2002a: 898 [2002b: 424]. TYPE LOCALITY: Israel [H in TAU].

DISTRIBUTION: Palaearctic: Israel.

fulva

Phthiria fulva Meigen, 1804.

Distribution: delete: Palaearctic: Belgium.

gaedii

Phthiria gaedii Wiedemann, 1820.

DistRIBUTION: add: Palaearctic: Cyprus, France (Corsica), Greece (Lefkas), Italy (Sardinia), Yugoslavia; delete: Palaearctic: Lithuania.

\section{lacteipennis}

Phthiria lacteipennis Strobl in Czerny \& Strobl, 1909.

DISTRIBUTION: add: Palaearctic: France.

\section{nitidigena}

Phthiria nitidigena Hesse, 1975.

DISTRIBUTION: add: Afrotropical: Namibia.

\section{pulicaria}

Bombylius pulicarius Mikan, 1796. 
Distribution: add: Palaearctic: Croatia, Czech Republic, Italy (Sicily), Spain

(Balearic Islands); delete: Palaearctic: Switzerland.

pulla ssp. pulla

Phthiria pulla Bezzi, 1922.

DISTRIBUTION: add: Afrotropical: Namibia.

socotrae

Phthiria socotrae Greathead \& Evenhuis, 2001a: 7. TYPE LOCALITY: Yemen (Sokotra) [H in NMSA*].

DISTRIBUTION: Afrotropical: Yemen (Sokotra).

subnitens

Phthiria subnitens Loew, 1846.

DistRIBUTION: add: Palaearctic: France, Greece (Crete), Italy (Sardinia), Macedonia, Spain.

umbripennis

Phthiria umbripennis Loew, 1846.

DisTRIBUTION: add: Palaearctic: Cyprus, Greece (Crete), Italy (Sardinia), Portugal.

vagans

Phthiria vagans Loew, 1846.

DisTRIBUTION: add: Palaearctic: Greece (Corfu, Crete, Kephalonia); delete:

Palaearctic: Lithuania.

Subfamily TOXOPHORINAE Schiner

Tribe GERONTINI Hesse

\section{albidipennis}

Geron albidipennis Loew, 1870.

DisTRIBUTION: add: Nearctic: Mexico (Sonora).

albipilosus

Geron albipilosus Hall \& Evenhuis, 2003: 17. TYPE LOCALITY: USA (New Mexico)

[H in USNM*].

DistRIBUTION: Nearctic: USA (California, Colorado, Nevada, New Mexico,

Utah). 
arenicola

Geron arenicola Painter, 1932.

DISTRIBUTION: add: Nearctic: USA (Nevada).

\section{argutus}

Geron argutus Painter, 1932.

Geron perplexus Tabet, 2001c: 49. TYPE LOCALITY: USA (Idaho) [H in CAS*] (new synonymy in Hall \& Evenhuis, 2003: 20).

asiaticus

Geron asiaticus Zaitzev, 1967.

Geron paramonovi Greathead, 2001b: 166. TYPE LOCALITY: Ukraine [H in ZMHB*]. [Preoccupied by Evenhuis, 1979.] SYN. N.

DISTRIBUTION: add: Palaearctic: Armenia, Ukraine.

\section{borealis}

Geron borealis Hall \& Evenhuis, 2003: 23. TYPE LOCALITY: USA (Nevada) [H in USNM*].

DISTRIBUTION: Nearctic: USA (Nevada).

\section{confusus}

Geron confusus Hall \& Evenhuis, 2003: 24. TYPE LOCALITY: USA (Arizona) [H in FSCA*].

DisTRIBUTION: Nearctic: USA (Arizona, New Mexico, Texas).

\section{cressoni}

Geron cressoni Hall \& Evenhuis, 2003: 26. TYPE LOCALITY: USA (Colorado) [H in USNM*].

DISTRIBUTION: Nearctic: USA (Arizona, Colorado, New Mexico, Washington)

elachys

Geron elachys Hall \& Evenhuis, 2003: 27. TYPE LOCALITY: USA (California) [H in USNM*].

DISTRIBUTION: Nearctic: USA (Arizona, California, Nevada).

eriogonae

Geron eriogonae Hall \& Evenhuis, 2003: 28. TYPE LOCALITY: USA (California) [H in $\left.\mathrm{USNM}^{*}\right]$

Distribution: Nearctic: Mexico (San Luis Potosí, Sonora, Zacatecas), USA (Arizona, California, Colorado, Nevada, New Mexico, Utah, Washington, Wyoming). 
Geron gibbosus var. erythropus Bezzi, 1925.

DISTRIBUTION: add: Palaearctic: Jordan.

furcifer

Geron furcifer Hesse, 1938.

DISTRIBUTION: add: Afrotropical: Namibia.

gibbosus

Geron gibbosus Olivier, 1789.

DistRIBUTION: add: Palaearctic: Greece (Rhodes, Thasos), Israel, ?Spain, Turkey.

halli

Geron halli Evenhuis in Hall \& Evenhuis, 2003: 32. TYPE LOCALITY: USA (Missouri) [H in USNM*].

DISTRIBUTION: Nearctic: USA (Missouri).

halteralis

Geron halteralis Wiedemann in Meigen, 1820.

DISTRIBUTION: add: Palaearctic: Italy (Sardinia)

\section{holosericeus}

Geron holosericeus Walker, 1849.

Geron saxosus Tabet, 1979 (synonymy in Hall \& Evenhuis, 2003: 33).

Geron saxosus Tabet, 2001c: 51. TYPE LOCALITY: USA (Idaho) [H in CAS*] (synonymy in Hall \& Evenhuis, 2003: 33).

DistRIBUTION: add: Nearctic: Mexico (Colima, Durango, Morelos). Neotropical:

El Salvador, Guatemala, Nicaragua.

infrequens

Geron infrequens Hall \& Evenhuis, 2003: 35. TYPE LOCALITY: USA (California) [H in $\mathrm{USNM}^{*}$.

DISTRIBUTION: Nearctic: USA (California).

kaszabi

Geron kaszabi Zaitzev, 1972.

DISTRIBUTION: add: Palaearctic: Uzbekistan.

kozlovi

Geron kozlovi Zaitzev, 1972.

DistRIBUTION: add: Palaearctic: Iran, Turkey, Uzbekistan. 
krymensis

Geron krymensis Paramonov, 1929.

DisTRIBUTION: add: Palaearctic: Jordan; delete: Palaearctic: Hungary.

macquarti

Geron macquarti Greathead in Evenhuis \& Greathead, 1999.

DistRIBUTION: add: Palaearctic: Turkey.

meigeni

Geron meigeni Greathead, 2001b: 170. TYPE LOCALITY: Italy (Sardinia) [H in ZMAN*].

DISTRIBUTION: Palaearctic: Algeria, Italy (Sardinia).

michaili

Geron michaili Zaitzev, 1972.

DistRIBUTION: add: Palaearctic: Algeria, Tunisia, Turkmenistan, Ukraine.

monticola

Geron monticola Hall \& Evenhuis, 2003: 37. TYPE LOCALITY: USA (California) [H in USNM*].

DISTRIBUTION: Nearctic: USA (California).

mystacinus

Geron mystacinus Bezzi, 1924.

DISTRIBUTION: Spain now confirmed (i.e.; delete “?”).

nevadensis

Geron nevadensis Hall \& Evenhuis, 2003: 40. TYPE LOCALITY: USA (Nevada) [H in $\mathrm{USNM}^{*}$.

DistRIBUTION: Nearctic: Mexico (Morelos), USA (Arizona, California, Nevada, Utah).

notios

Geron notios Hall \& Evenhuis, 2003: 44. TYPE LOCALITY: USA (Arizona) [H in

CAS*].

DISTRIBUTION: Nearctic: Mexico (Nayarít, Sonora), USA (Arizona, California, Texas).

olivierii

Geron olivierii Macquart, 1840.

Distribution: add: Palaearctic: Armenia, Croatia, Greece (Kephalonia, Naxos, Poros, Rhodes), Italy, Jordan, Spain, Turkmenistan. 
Geron painteri Hall \& Evenhuis, 2003: 46. TYPE LOCALITY: USA (Arizona) [H in USNM*].

DisTRIBUTION: Nearctic: USA (Arizona, California, New Mexico).

peucon

Geron peucon Hall \& Evenhuis, 2003: 48. TYPE LOCALITY: USA (California) [H in USNM*].

DistRIBUTION: Nearctic: USA (Arizona, California, Idaho, Nevada, Utah).

prosopidis

Geron prosopidis Hall \& Evenhuis, 2003: 50. TYPE LOCALITY: USA (California) [H in USNM*].

DisTRIBUTION: Nearctic: Canada (British Columbia), Mexico (Nuevo León), USA (Arizona, California, Colorado, Kansas, Nevada, Utah, Washington, Wyoming).

\section{psammobates}

Geron psammobates Hesse, 1938.

DisTRIBUTION: add: Afrotropical: Namibia.

senilis

Geron senilis Fabricius, 1794.

Geron antologos Tabet, 2001c: 48. TyPE LOCALITY: USA (Idaho) [H in CAS*] (new synonymy in Hall \& Evenhuis, 2003: 55).

DistRIBUTION: add: Nearctic: Mexico (Durango, San Luis Potosí, VeracruzLlave), USA (Illinois, Maryland, New Jersey, Virginia). Neotropical: Mexico (Oaxaca).

smirnovi

Geron smirnovi Zaitzev, 1978.

DisTRIBUTION: add: Palaearctic: Jordan, Turkey.

stenos

Geron stenos Hall \& Evenhuis, 2003: 58. TYPE LOCALITY: USA (California) [H in USNM*]

DISTRIBUTION: Nearctic: USA (California).

\section{subauratus}

Geron subauratus Loew, 1863.

DISTRIBUTION: add: Nearctic: USA (New York, Virginia). 
syriacus

Geron syriacus Zaitzev, 2002c: 439. TYPE LOCALITY: Lebanon [H in NHRS].

DISTRIBUTION: Palaearctic: Lebanon.

weemsi

Geron weemsi Hall \& Evenhuis, 2003: 65. TYPE LOCALITY: USA (Florida) [H in FSCA*].

DISTRIBUTION: Nearctic: USA (Florida).

Tribe SYSTROPODINI Brauer

Genus SYSTROPUS Wiedemann

liuae

Systropus liuae Nagatomi, Tamaki \& Evenhuis, 2000: 16. TYPE LOCALITY: Japan [H in NSMT*].

Distribution: Oriental: Taiwan. Palaearctic: Japan.

Tribe TOXOPHORINI Schiner

Genus TOXOPHORA Meigen

australis

Toxophora australis Hesse, 1938.

DisTRIBUTION: add: Afrotropical: Namibia.

\section{fasciculata}

Asilus fasciculatus Villers, 1789.

Distribution: add: Palaearctic: Greece (Crete, Ithaca, Rhodes); delete: Palaearctic: Czech Republic, Germany, Slovakia, Switzerland.

gittinsi

Toxophora gittinsi Tabet, 1979.

Toxophora gittinsi Tabet, 2001a: 44. TYPE LOCALITY: USA (Idaho) [H in CAS]. DISTRIBUTION: Nearctic: USA (Idaho).

\section{obliquisquamosa}

Toxophora obliquesquamosa Hesse, 1938.

DisTRIBUTION: add: Afrotropical: Namibia. 
Toxophora quadricellulata Hesse, 1938.

DISTRIBUTION: add: Afrotropical: Namibia.

\title{
Subfamily HETEROTROPINAE Becker
}

\section{Genus HETEROTROPUS Loew}

apertus

Heterotropus apertus Greathead, 2000: 57. TYPE LOCALITY: Namibia [H in NMNW*]. DISTRIBUTION: Afrotropical: Namibia.

israeliensis

Heterotropus israeliensis Zaitzev, 2002a: 896 [2002b: 426]. TYPE LOCALITY: Israel [H in TAU].

DISTRIBUTION: Palaearctic: Israel.

\section{kaplanae}

Heterotropus kaplanae Zaitzev, 2002a: 898 [2002b: 428]. TYPE LOCALITY: Israel [H in TAU]

DisTRIBUTION: Palaearctic: Israel.

taurus

Heterotropus taurus Bowden, 1967.

DistRIBUTION: add: Palaearctic: Greece (Rhodes).

\author{
Subfamily BOMBYLIINAE Latreille \\ Tribe BOMBYLIINI Latreille
}

Genus ANASTOECHUS Osten Sacken

bahirae

Anastoechus bahirae Becker, 1915.

DISTRIBUTION: add: Palaearctic: Jordan.

exalbidus

Bombylius exalbidus Wiedemann in Meigen, 1820

DisTRIBUTION: add: Palaearctic: France (Corsica). 


\section{fulvescens}

Anastoechus fulvescens Becker in Becker \& Stein, 1913.

DISTRIBUTION: add: Palaearctic: Spain.

\section{hyrcanus}

Bombylius hyrcanus Pallas \& Wiedemann in Wiedemann, 1818.

DistRIBUTION: add: Palaearctic: ?Azores, Greece (Poros).

\section{innocuus}

Anastoechus innocuus Bezzi, 1921.

DISTRIBUTION: add: Afrotropical: Namibia.

\section{leucosoma}

Anastoechus leucosoma Bezzi, 1921.

DisTRIBUTION: add: Afrotropical: Namibia.

\section{nitidulus}

Anastoechus nitidulus Fabricius, 1794.

Anastoechus olivaceus Paramonov, 1930. SYN. N.

Anastoechus olivaceus var. corsikanus Paramonov, 1930. SYN. N.

Anastoechus olivaceus var. corsicanus Zaitzev, 1989. SYN. N.

Distribution: add: Palaearctic: Cyprus, France (Corsica), Finland, Greece

(Rhodes), Jordan, Poland, Ukraine; delete: Palaearctic: Belgium.

\section{varipecten}

Anastoechus varipecten Bezzi, 1921.

Anastoechus leucochroicus Hesse, 1938: 373. SYN. N.

DisTRIBUTION: add: Afrotropical: Namibia, South Africa (Western Cape).

\section{Genus AUSTRALOECHUS Greathead}

\section{naibensis}

Bombylius naibenis, Hesse, 1961.

DisTRIBUTION: add: Afrotropical: Namibia.

peringueyi

Bombylius peringueyi Bezzi, 1921.

DISTRIBUTION: add: Afrotropical: Namibia.

\section{punctifer}

Bombylius punctifer Bezzi, 1921.

DISTRIBUTION: add: Afrotropical: Namibia. 
androgynus

Bombylius androgynus Loew, 1855.

Distribution: add: Palaearctic: Greece (Corfu, Ithaca, Kephalonia).

\section{Genus BOMBOMYIA Greathead}

\section{discoidea}

Bombylius discoideus Fabricius, 1784.

DistrIBUTION: add: Palaearctic: Bulgaria,

\section{vertebralis}

Bombylius punctatus Fabricius, 1794. SYN. N.

Bombilius vertebralis Dufour, 1833.

Bombylius sticticus Boisduval, 1835. SYN. N.

Bombylius subluna Walker, 1849. SYN. N.

Distribution: Palaearctic: Afghanistan, Albania, Algeria, Armenia, Austria, Azerbaijan, Bosnia-Herzegovina, Croatia, Czech Republic, Egypt, France, Gibraltar, Greece (incl. Corfu, Lesbos), Gruzia, Hungary, Iran, Iraq, Israel, Italy (incl. Sardinia, Sicily), Kazakhstan, Kuwait, Kyrgyz Republic, Lebanon, Libya, Moldova, Morocco, Portugal, Romania, Russia (SET), Spain, Syria, Tajikistan, Tunisia, Turkey, Turkmenistan, Ukraine, United Arab Emirates

\section{Genus BOMBYLELLA Greathead}

atra

Bombylius ater Scopoli, 1763.

DistRIBUTION: add: Palaearctic: France (Corsica), Germany, Greece (Corfu, Lefkas), Jordan, Moldova, Portugal; delete: Palaearctic: Lithuania.

elegans

Bombylius elegans Wiedemann, 1828.

DISTRIBUTION: add: Afrotropical: South Africa (Kwazulu-Natal, Limpopo)

\section{rufiventris}

Bombylius rufiventris Macquart, 1846.

DISTRIBUTION: add: Afrotropical: Tanzania.

\section{simulans}

Bombylius simulans Austen, 1937.

DISTRIBUTION: add: Palaearctic: Jordan. 
album

Bombylisoma album Greathead, 2000: 59. TYPE LOCALITY: Namibia [H in NMNW*]. DISTRIBUTION: Afrotropical: Namibia.

\section{breviusculum}

Bombylius breviusculus Loew, 1855.

Distribution: delete: Palaearctic: Germany, Poland.

\section{croaticum}

Bombylius croaticus Kertész, 1901.

Distribution: delete: Palaearctic: Czech Republic, Germany, Poland, Slovakia, Switzerland.

\section{flavibarbum}

Bombylius flavibarbus Loew, 1855.

Distribution: delete: Palaearctic: Spain.

\section{melanocephalum}

Bombylius melanocephalus Fabricius, 1794.

Distribution: delete: Palaearctic: Czech Republic, Hungary, Slovakia, Switzerland.

minimum

Bombylius minimus Scopoli, 1771.

Distribution: delete: Palaearctic: Germany.

nigriceps

Dischistus nigriceps Loew, 1862.

Distribution: delete: Palaearctic: Switzerland.

nucale

Dischistus nucalis Bezzi, 1924.

DistRIBUTION: add: Afrotropical: Namibia.

unicolor

Bombylius unicolor Loew, 1855.

Distribution: add: Palaearctic: Austria, Portugal. 
aksarayensis

Bombylius aksarayensis Hasbenli \& Zaitzev, 2000: 105. TYPE LOCALITY: Turkey [H in ZMGU].

Distribution: Palaearctic: Turkey.

ambustus

Bombylius ambustus Pallas \& Wiedemann in Wiedemann, 1818.

Distribution: delete: Palaearctic: Czech Republic, Germany, Lithuania, Slovakia, Switzerland.

analis ssp. analis

Bombylius analis Olivier, 1789.

Distribution: delete: Palaearctic: Lithuania, Switzerland.

candidus

Bombylius candidus Loew, 1855.

DistRIBUTION: delete: Palaearctic: Germany.

canescens

Bombylius canescens Mikan, 1796.

DisTRIBUTION: add: Palaearctic: Andorra, Greece (Crete, Ithaca), Jordan; delete:

Palaearctic: Lithuania, UK (Northern Ireland).

cinerascens

Bombylius cinerascens Mikan, 1796.

DistRIBUTION: add: Palaearctic: Andorra, Cyprus, France (Corsica), Greece (Crete, Rhodes), Jordan, Portugal, Spain (Isla Cabrera).

citrinus

Bombylius citrinus Loew, 1855.

Distribution: delete: Palaearctic: Germany, Poland

discolor

Bombylius discolor Mikan, 1796.

DisTRIBUTION: add: Palaearctic: Greece (Crete), Netherlands.

fimbriatus ssp. fimbriatus

Bombylius fimbriatus Meigen, 1820.

Distribution: add: Palaearctic: Bosnia-Herzegovina, Greece (Crete, Lesbos), 


\section{fulvescens}

Bombylius fulvescens Wiedemann in Meigen, 1820.

Distribution: add: Palaearctic: Egypt.

\section{fuscus}

Bombylius fuscus Fabricius, 1781.

DistRIBUTION: add: Palaearctic: Croatia, France, Jordan.

major

Bombylius major Linnaeus, 1758.

DisTRIBUTION: add: Palaearctic: Jordan; delete: Palaearctic: UK (Northern Ireland).

medius

Bombylius medius Linnaeus, 1758.

DistRIBUTION: add: Palaearctic: Greece (Corfu, Crete, Lesbos), Jordan, Norway.

\section{mendax}

Bombylius mendax Austen, 1937.

DisTRIBUTION: add: Palaearctic: Jordan.

minor

Bombylius minor Linnaeus, 1758.

Bombylius flavescens Palm, 1876. SYN. N.

Bombylius minor var. ochraceus Paramonov, 1926. SYN. N.

Bombylius luteolus Evenhuis, 1978. SYN. N.

Bombylius thapsinoides Evenhuis, 1978. SYN. N.

DistRIBUTION: add: Palaearctic: Croatia, France (Corsica), Greece (Corfu, Kephalonia, Lefkas, Poros, Rhodes); delete: Palaearctic: Lithuania, Netherlands, Switzerland.

modestus

Bombylius modestus Loew 1873.

Distribution: add: Palaearctic: Greece (Corfu, Poros, Rhodes, Zakynthos), Jordan.

mus

Bombylius mus Bigot, 1862.

Distribution: add: Palaearctic: Spain (Balearic Islands); delete: Palaearctic:

Hungary. 
Distribution: add: Palaearctic: Croatia, Greece (Poros).

nubilus ssp. nubilus

Bombylius nubilus Mikan, 1796.

DistRIBUTION: add: Palaearctic: Turkey.

pallens

Bombylius pallens Wiedemann, 1820.

Distribution: add: Palaearctic: Spain (Balearic Islands).

posticus

Bombylius posticus Fabricius, 1805.

Distribution: add: Palaearctic: Greece (Corfu, Crete, Ithaca, Rhodes, Santorini, Zakynthos), Jordan, Lithuania.

pumilus

Bombylius pumilus Meigen, 1820.

Distribution: add: Palaearctic: France (Corsica), Greece (Ithaca, Lesbos), Jordan; delete: Palaearctic: Germany, Poland.

\section{semifuscus}

Bombylius semifuscus Meigen, 1820.

Distribution: delete: Palaearctic: Germany, Slovakia.

\section{spinulosus}

Bombylius spinulosus Hasbenli \& Zaitzev, 2000: 106. TYPE LOCALITY: Turkey [H in ZMGU].

DistRIBUTION: Palaearctic: Turkey.

\section{trichurus}

Bombylius trichurus Pallas in Wiedemann, 1818.

Bombylius rhodius Loew, 1855. SYN. N.

DistRIBUTION: add: Palaearctic: Jordan, Yugoslavia (Montenegro); delete:

Palaearctic: Czech Republic, Slovakia.

venosus

Bombylius venosus Mikan 1796.

Distribution: add: Palaearctic: Spain (Mallorca); delete: Palaearctic: Lithuania. 


\section{cinerarius}

Bombylius cinerarius Pallas \& Wiedemann in Wiedemann, 1818.

DistRIBUTION: add: Palaearctic: France (Corsica); delete: Palaearctic: Hungary, Slovakia.

\section{cruciatus}

Bombylius cruciatus Fabricius, 1798.

Distribution add: Palaearctic: France (Corsica), Greece (Crete), Portugal; delete: Palaearctic: Belgium, Poland.

\section{incanus}

Bombylius incanus Johnson, 1907.

DISTRIBUTION: add: Nearctic: USA (Connecticut, Georgia).

\section{quadrifarius}

Bombylius quadrifarius Loew, 1855.

DISTRIBUTION: add: Palaearctic: Jordan.

\section{Genus DISCHISTUS Loew}

syriacus

Bombylius syriacus Villeneuve, 1912.

DISTRIBUTION: add: Palaearctic: Jordan.

\section{Genus EUPREPINA Hull}

nuda

Euprepina nuda Hull, 1971.

DisTRIBUTION: add: Neotropical: Brazil (Minas Gerais).

\section{Genus GONARTHRUS Bezzi}

\section{chloroxanthus}

Gonarthrus chloroxanthus Hesse, 1938.

DISTRIBUTION: add: Afrotropical: Namibia. 
croceum

Heterostylum croceum Painter, 1930.

DISTRIBUTION: add: Nearctic: USA (Illinois).

Genus NEOBOMBYLODES Evenhuis

giganteus

Dischistus giganteus Villeneuve, 1920.

DISTRIBUTION: add: Palaearctic: Jordan.

\section{Genus SYSTOECHUS Loew}

autumnalis

Bombylius autumnalis Pallas \& Wiedemann in Wiedemann, 1818.

Distribution: delete: Palaearctic: Czech Republic, Hungary, Slovakia.

\section{ctenopterus}

Bombylius ctenopterus Mikan, 1796.

Bombylius aurulentus Wiedemann in Meigen, 1820. SYN. N.

Distribution: add: Palaearctic: Bosnia-Herzegovina, Egypt, France (Corsica), Greece (Corfu , Crete), Italy (Sardinia); delete: Palaearctic: Netherlands.

\section{deceptus}

Systoechus deceptus Hesse, 1938.

DisTRIBUTION: add: Afrotropical: Namibia.

\section{gomezmenori}

Systoechus gomezmenori Andréu Rubio, 1959.

Distribution: delete: Palaearctic: Germany, Spain.

\section{gradatus}

Bombylius gradatus Wiedemann in Meigen, 1820.

Bombylius lucidus Loew, 1855. SYN. N.

Systoechus tesquorum Becker, 1916. SYN. N.

DistRIBUTION: add: Palaearctic: Afghanistan, France (Corsica), Kazakhstan, Macedonia, Moldova, Morocco, Poland, Russia (SET), Tunisia, Turkey, Ukraine; delete: Palaearctic: Germany. 
Systoechus longirostris Becker, 1916.

DistRIBUTION: add: Palaearctic: Jordan; delete: Palaearctic: Germany.

\section{microcephalus}

Bombylius microcephalus Loew, 1855.

DISTRIBUTION: add: Palaearctic: Spain.

\section{Genus TRIPLASIUS Loew}

pictus

Bombylius pictus Panzer, 1794.

Distribution: add: Palaearctic: Bosnia-Herzegovina, Croatia, Slovenia.

\section{Tribe CONOPHORINI Becker}

Genus CONOPHORUS Meigen

\section{fuliginosus}

Ploas fuliginosus Wiedemann, 1820.

DisTRIBUTION: add: Palaearctic: Gibraltar.

glaucescens

Ploas glaucescens Loew, 1863.

DisTRIBUTION: add: Palaearctic: Greece (Lesbos), Jordan.

griseus

Bombylius griseus Fabricius, 1787.

Distribution: add: Palaearctic: Portugal.

\section{macroglossus}

Ploas macroglossa Dufour, 1852.

DISTRIBUTION: add: Palaearctic: France.

nobilis

Ploas nobilis Loew, 1873.

DisTRIBUTION: add: Palaearctic: Greece (Lesbos), Jordan.

pusillus

Ploas pusillus Loew, 1869. 
rossicus

Conophorus rossicus Paramonov, 1929.

DistRIBUTION: delete: Palaearctic: Czech Republic, Slovakia.

virescens

Bombylius virescens Fabricius, 1789.

DistRIBUTION: add: Palaearctic: Bulgaria, Croatia, Greece (Corfu), Macedonia, Romania, Slovenia; delete: Palaearctic: Lithuania.

\section{Genus LEGNOTOMYIA Bezzi}

\section{cineracea}

Legnotomyia cineracea Austen, 1937.

DistRIBUTION: add: Palaearctic: Jordan.

\section{trichorhoea}

Bombylius trichoroeus Loew, 1855.

DISTRIBUTION: add: Palaearctic: Jordan.

\section{Genus NOTOLEGNOTUS Greathead \& Evenhuis}

NOTOLEGNOTUS Greathead \& Evenhuis, 2001b: 163. Type species Notolegnotus brevis Greathead \& Evenhuis, 2001, by original designation.

brevis

Notolegnotus brevis Greathead \& Evenhuis, 2001b: 164. TYPE LOCALITY: Namibia [H in NMSA].

DISTRIBUTION: Afrotropical: Namibia.

Subfamily ECLIMINAE Hall

Genus ECLIMUS Loew

gracilis

Eclimus gracilis Loew, 1844.

Distribution: add: Palaearctic: Greece (Kephalonia, Ithaca, Lesbos). 


\title{
cuthbertsoni
}

Paratoxophora cuthbertsoni Engel, 1936.

DISTRIBUTION: add: Afrotropical: Namibia.

\author{
Subfamily CROCIDIINAE Hull \\ Genus APATOMYZA Wiedemann
}

APATOMYZA Wiedemann, 1820.

Adelogenys Hesse, 1938: 811. Type species Adelogenys culicoides Hesse, 1938. (synonymy by Lamas, Evenhuis \& Couri, 2001: 176.)

aurea

Apatomyza aurea Lamas, Evenhuis \& Couri, 2003: 101. TYPE LOCALITY: South Africa (Western Cape) [H in NMSA*].

DistRIBUTION: Afrotropical: South Africa (Western Cape).

\section{bethuliensis}

Apatomyza bethuliensis Lamas, Evenhuis \& Couri, 2003: 97. TYPE LOCALITY: South Africa (Free State) [H in NMSA*].

Distribution: Afrotropical: South Africa (Free State).

\section{braunsi}

Adelogenys braunsi Hesse, 1938 (new combination in Lamas, Evenhuis \& Couri, 2001: 178).

DisTRIBUTION: add: Afrotropical: South Africa (Northern Cape, Western Cape).

capensis

Apatomyza capensis Lamas, Evenhuis \& Couri, 2001: 178. TYPE LOCALITY: South Africa (Western Cape) [H in NMSA*].

DisTRIBUTION: Afrotropical: South Africa (Western Cape).

\section{culicoides}

Adelogenys culicoides Hesse, 1938 (new combination in Lamas, Evenhuis \& Couri, 2001: 180).

\section{desertica}

Apatomyza desertica Lamas, Evenhuis \& Couri, 2001: 182. TYPE LOCALITY: Namibia [H in NMSA*].

DisTRIBUTION: Afrotropical: Namibia. 
Apatomyza glabripleura Lamas, Evenhuis \& Couri, 2003: 103. TYPE LOCALITY: South Africa (Western Cape) [H in NMSA*].

DistRIBUTION: Afrotropical: South Africa (Western Cape).

irwini

Apatomyza irwini Lamas, Evenhuis \& Couri, 2001: 183. TyPE LOCALITY: South Africa (Northern Cape) [H in NMSA*].

DistRIBUTION: Afrotropical: South Africa (Northern Cape), Namibia.

proboscidea

Apatomyza proboscidea Lamas, Evenhuis \& Couri, 2003: 99. TYPE LOCALITY: South Africa (Free State) [H in NMSA*].

DistRIBUTION: Afrotropical: South Africa (Eastern Cape, Free State).

\section{punctipennis}

Apatomyza punctipennis Wiedemann, 1820.

Adelogenys namaquaensis Hesse, 1938 (new synonymy in Lamas, Evenhuis \& Couri, 2001: 185).

Distribution: add: Afrotropical: Namibia, South Africa (Northern Cape).

\section{Genus CROCIDIUM Loew}

\section{dichopticum}

Crocidium dichopticum Hesse, 1963.

DISTRIBUTION: add: Afrotropical: Namibia.

\section{fuscum}

Crocidium fuscum Greathead, 2000: 60. TYPE LOCALITY: Namibia [H in NMNW*]

DisTRIBUTION: Afrotropical: Namibia.

\section{giganteum}

Crocidium giganteum Lamas, Evenhuis \& Couri, 2003: 104. TYPE LOCALITY: South Africa (Northern Cape) [H in NMSA*].

DistRIBUTION: Afrotropical: South Africa (Northern Cape).

\section{globithorax}

Crocidium globithorax Lamas, Evenhuis \& Couri, 2003: 108. TYPE LOCALITY: South Africa (Northern Cape) [H in NMSA*].

DistRIBUTION: Afrotropical: South Africa (Northern Cape). 
hessei

Crocidium hessei Lamas, Evenhuis \& Couri, 2003: 106. TyPE LOCALITY: South Africa (Northern Cape) [H in NMSA*].

DistRIBUTION: Afrotropical: South Africa (Northern Cape).

immaculatum

Crocidium immaculatum Bezzi, 1922.

Distribution: add: Afrotropical: Namibia.

pallens

Crocidium pallens Greathead, 2000: 61. TYPE LOCALITY: Namibia [H in NMNW*]

DISTRIBUTION: Afrotropical: Namibia.

\section{Subfamily MARIOBEZZIINAE Becker \\ Genus CORSOMYZA Wiedemann}

\section{eremobia}

Corsomyza eremobia Hesse, 1938.

DISTRIBUTION: add: Afrotropical: Namibia.

\section{Genus HYPERUSIA Bezzi}

apiformis

Hyperusia apiformis Greathead \& Evenhuis, 2001b: 170. TYPE LOCALITY: Tanzania [H in BMNH*].

DisTRIBUTION: Afrotropical: Tanzania.

Genus MEGAPALPUS Macquart

capensis

Phthiria capensis Wiedemann, 1828.

DISTRIBUTION: add: Afrotropical: Namibia.

Subfamily CYTHEREINAE Becker

Genus AMICTUS Wiedemann

minor

Amictus minor Austen, 1937. 
obliquenotatus

Amictus obliquenotatus Austen, 1937.

Distribution: add: Palaearctic: Jordan.

pictus

Amictus pictus Loew, 1869.

Distribution: add: Palaearctic: Greece (Crete, Ithaca, Kephalonia, Rhodes)

pulchellus

Amictus pulchellus Macquart, 1846.

DistRIBUTION: add: Palaearctic: Macedonia.

strigilatus

Amictus strigilatus Loew, 1869. STAT. REV. (removed from synonymy under Amictus pulchellus Macquart).

DISTRIBUTION: Palaearctic: Greece (incl. Rhodes), ?Israel, Turkey.

validus

Amictus validus Loew, 1869.

DisTRIBUTION: add: Palaearctic: Greece (Lesbos), Jordan.

virgatus

Amictus virgatus Austen, 1937.

DISTRIBUTION: add: Palaearctic: Jordan.

Genus CALLOSTOMA Macquart

fascipenne

Callostoma fascipennis Macquart, 1840.

DisTRIBUTION: add: Palaearctic: Jordan.

Genus CHALCOCHITON Loew

carmelitensis

Mulio carmelitensis Becker, 1903. COMB. N. (moved to Chalcochiton from Cytherea).

pallasii

Mulio pallasii Loew, 1856.

Distribution: add: Palaearctic: Jordan; delete: Palaearctic: Poland. 
speciosus

Chalcochiton speciosus Loew, 1844.

DISTRIBUTION: add: Palaearctic: Jordan.

syriacus

Mulio syriacus Loew, 1869.

DISTRIBUTION: add: Palaearctic: Jordan.

\title{
Genus CYLLENIA Latreille
}

marginata

Cyllenia marginata Loew, 1846.

DisTRIBUTION: add: Palaearctic: Greece (Ithaca), Italy (Sardinia).

rustica

Asilus rusticus Rossi, 1790.

Distribution: add: Palaearctic: France (Corsica), Greece (Corfu, Crete,

Rhodes); delete: Palaearctic: Czech Republic, Germany, Poland, Slovakia, Switzerland.

\section{Genus CYTHEREA Fabricius}

aurea

Cytherea aurea Fabricius, 1794. STAT. REV. (moved from Chalcochiton back to Cytherea).

\section{fenestrata}

Mulio fenestrata Loew, 1873.

DISTRIBUTION: add: Palaearctic: Jordan.

obscura

Cytherea obscura Fabricius, 1794.

DistRIBUTION: add: Palaearctic: Greece (Corfu, Ithaca, Kephalonia, Rhodes), Jordan; delete: Palaearctic: Czech Republic, Slovakia.

\author{
Subfamily LOMATIINAE Schiner
}

Genus LOMATIA Meigen

\section{abbreviata}

Lomatia abbreviata Villeneuve, 1911. 
alecto

Lomatia alecto Loew, 1846.

Distribution: delete: Palaearctic: Czech Republic, Slovakia.

atropos

Lomatia atropos Egger, 1859.

DistRIBUTION: add: Palaearctic: Greece (Corfu).

belzebul

Anthrax belzebul Fabricius, 1794.

Distribution: add: Palaearctic: Portugal, Switzerland.

erynnis

Lomatia erynnis Loew, 1869.

DistRIBUTION: add: Palaearctic: Greece (Corfu, Euboea, Kephalonia, Skyros); delete: Palaearctic: Spain.

grajugena

Lomatia grajugena Loew, 1869.

DistRIBUTION: add: Palaearctic: Greece (Euboea, Poros).

lachesis

Lomatia lachesis Egger, 1859.

DistRIBUTION: delete: Palaearctic: Switzerland.

polyzona

Lomatia polyzona Loew, 1869.

DISTRIBUTION: add: Palaearctic: Jordan.

\section{rogenhoferi}

Lomatia rogenhoferi Nowicki, 1868.

DistRIBUTION: add: Palaearctic: Czech Republic, Slovakia.

\section{tysiphone}

Lomatia tysiphone Loew in Schiner, 1860.

DistRIBUTION: add: Palaearctic: Greece (Kephalonia), Jordan, Portugal 


\title{
leucoprocta
}

Mulio leucoprocta Wiedemann, 1828.

DISTRIBUTION: add: Nearctic: USA (Connecticut).

\author{
Subfamily ANTONIINAE Hull
}

Genus ANTONIA Loew

\section{xanthogramma}

Antonia xanthogramma Bezzi, 1924.

DisTRIBUTION: add: Afrotropical: Namibia.

\section{Subfamily ANTHRACINAE Latreille}

Tribe ANTHRACINI Latreille

\section{Genus ANTHRAX Scopoli}

aethiops ssp. aethiops

Bibio aethiops Fabricius, 1781.

DistRIBUTION: add: Palaearctic: Greece (Rhodes), Jordan, Macedonia, Moldova.

aethiops ssp. bezzii

Spogostylum aethiops bezzii Paramonov, 1957 [L designated by El Hawagry et al. (2000: 101) in ESEC].

\section{angustipennis}

Anthrax angustipennis Macquart, 1840.

DisTRIBUTION: add: Neotropical: Peru.

\section{anthrax}

Musca anthrax Schrank, 1781.

Distribution: add: Palaearctic: Bosnia-Herzegovina, Madeira, Spain (Balearic Islands).

aterrimus

Argyramoeba aterrima Bigot, 1892. 


\section{candidapex}

Spogostylum candidapex Austen, 1937.

DISTRIBUTION: add: Palaearctic: Egypt. Jordan.

\section{chionanthrax}

Argyramoeba chionanthrax Bezzi, 1926.

Anthrax cairensis Paramonov, 1935 [H found! (by El Hawagry et al. (2000: 102) in ESEC].

Anthrax nanus Paramonov, 1935 [L designated by El Hawagry et al. (2000: 102) in ESEC].

\section{chionostigma}

Anthrax chionostigma Tsacas, 1962.

Argyramoeba maculosa Sack, 1909. SYN. N.

Anthrax ipiriensis Dils \& Weyer, 1995. SYN. N.

Anthrax tajikistanus Evenhuis \& Greathead, 1999. SYN. N.

DisTRIBUTION: add: Palaearctic: Afghanistan, Armenia, Azerbaijan, France (incl.

Corsica), Gruzia, Kazakhstan, Spain, Tajikistan, Uzbekistan.

\section{dentata}

Argyromoeba dentata Becker, 1906.

Anthrax efflatouni Paramonov, 1936 (synonymy in El Hawagry et al., 2000: 102).

DISTRIBUTION: revised: Palaearctic: ?Andorra, Egypt, Hungary, Israel (incl. West Bank), Italy (incl. Sicily), Jordan, Saudi Arabia, Spain, Tunisia.

\section{diffusus}

Anthrax diffusa Wiedemann, 1824.

DISTRIBUTION: add: Afrotropical: Namibia.

galali

Anthrax galali El Hawagry, 2002: 2. TYPE LOCALITY: Egypt [H in CUE].

DISTRIBUTION: Palaearctic: Egypt.

\section{greatheadi}

Anthrax greatheadi El Hawagry, 1999: 108. TYPE LOCALITY: Egypt [H in CUE].

DisTRIBUTION: Palaearctic: Egypt, Jordan.

\section{hyalacrus}

Anthrax hyalacrus Wiedemann, 1828.

DisTRIBUTION: add: Neotropical: Panama, Trinidad \& Tobago. 
johanni

Anthrax johanni Zaitzev, 1997.

DisTRIBUTION: add: Afrotropical: Chad, Eritrea, Kenya, Yemen. Palaearctic:

Israel, Malta, Saudi Arabia.

lucidus

Argyromoeba lucida Becker, 1902.

Anthrax hassani Paramonov, 1935 [L designated by El Hawagry et al. (2000: 103) in

ESEC]. (synonymy in El Hawagry et al., 2000: 103)

Spogostylum nigrum Austen, 1937. SYN. N.

DisTRIBUTION: add: Palaearctic: Israel (incl. West Bank), Tunisia.

melanista

Argyromoeba melanista Bezzi, 1925 [L designated by El Hawagry et al. (2000: 104)

in ESEC].

moursyi

Anthrax moursyi El Hawagry, 1999: 109. TYPE LOCALITY: Egypt [H in CUE].

DISTRIBUTION: Palaearctic: Egypt.

muticus

Spongostylum muticum Bezzi, 1921.

DISTRIBUTION: add: Afrotropical: Namibia.

phaeopteralis

Anthrax phaeopteralis Hesse, 1956.

Distribution: add: Afrotropical: Namibia.

pilosulus

Argyramoeba pilosulus Strobl, 1902

DisTRIBUTION: add: Palaearctic: Croatia, France, Greece (Corfu).

ricardoae

Argyromoeba fuscipennis Ricardo, 1903 [Preoccupied by Macquart, 1834.] (removed from synonymy with dentata by Greathead, 2003: 91).

Anthrax ricardoae Greathead, 2003: 91 (new replacement name for Anthrax fuscipennis Ricardo, 1903).

DISTRIBUTION: Afrotropical: Yemen (Sokotra).

sticticus

Anthrax stictica Klug, 1832.

DistRIBUTION: add: Palaearctic: Egypt, Jordan. 
Anthrax trifasciata Meigen, 1804.

Anthrax leucogaster Wiedemann in Meigen, 1820 (synonymy in Greathead, 2003: 92).

Argyramoeba heteropyga Sack, 1909 (synonymy in Greathead, 2003: 92).

DisTRIBUTION: add: Palaearctic: Belarus, Egypt, Finland, Germany, Greece

(Kephalonia, Poros, Rhodes), Italy (Sicily), Norway, Romania, Sweden,

Ukraine.

\section{trisinuatus}

Anthrax trisinuatus Hesse, 1956.

DisTRIBUTION: add: Afrotropical: Namibia.

varius

Anthrax varia Fabricius, 1794.

DISTRIBUTION: delete: Palaearctic: Canary Islands.

virgo

Anthrax virgo Egger, 1859.

Argyramoeba velox Loew, 1862a. SYN. N.

Anthrax hellenicus François, 1966. SYN. N.

DistRIBUTION: add: Palaearctic: Greece (Kephalonia, Poros), Jordan, Macedonia; delete Palaearctic: Hungary.

\section{zohrayensis}

Anthrax zohrayensis El Hawagry, 2002: 4. TYPE LOCALITY: Egypt [H in CUE].

DISTRIBUTION: Palaearctic: Egypt.

\section{Genus BRACHYANAX Evenhuis}

\section{satellitius}

Anthrax satellitia Walker, 1956.

DISTRIBUTION: add: Oriental: Singapore.

\section{Genus SPOGOSTYLUM Macquart}

\section{bisniphas}

Argyramoeba bisniphas Bezzi, 1925. [L designated by El Hawagry et al. (2000: 107) in ESEC]. (moved from Anthrax to Spogostylum in El Hawagry et al., 2000: 107). 
candidum

Psamatamoeba candida Sack, 1909.

DISTRIBUTION: add: Palaearctic: Jordan.

griseipenne

Exoprosopa griseipennis Macquart, 1850.

DISTRIBUTION: add: Palaearctic: Jordan.

hamadnallahi

Spogostylum hamadnallahi El Hawagry, 2002: 6. TYPE LOCALITY: Egypt [H in CUE]. DISTRIBUTION: Palaearctic: Egypt.

isis

Anthrax isis Meigen, 1820.

DistRIBUTION: add: Palaearctic: Greece (Rhodes), Jordan.

nitidum

Spogostylum nitidum Austen, 1937. COMB. REV. (returned to Spogostylum from Anthrax).

Distribution: add: Palaearctic: Jordan.

ocyale

Anthrax ocyale Wiedemann, 1828.

DISTRIBUTION: add: Palaearctic: United Arab Emirates.

perpusillum

Spogostylum perpusillum Austen, 1937.

DISTRIBUTION: add: Palaearctic: Jordan.

sordidum

Spogostylum sordidum Sack, 1909.

Spongostylum antiopa Bezzi, 1925 [H found! in ESEC] (synonymy in El Hawagry et al., 2000: 110).

DisTRIBUTION: add: Palaearctic: Jordan.

\section{tripunctatum}

Anthrax tripunctata Wiedemann in Meigen, 1820.

Distribution: add: Palaearctic: Greece (Kephalonia), Italy (Sardinia, Sicily), Portugal; delete: Palaearctic: Switzerland.

ventrale

Spongostylum ventrale Bezzi, 1924.

Distribution: add: Palaearctic: Egypt. 


\section{Genus APHOEBANTUS Loew}

idahoensis

Aphoebantus idahoensis Tabet, 1979.

Aphoebantus idahoensis Tabet, 2001b: 45. TYPE LOCALITY: USA (Idaho) [H in CAS]. DISTRIBUTION: Nearctic: USA (Idaho).

paraborealis

Aphoebantus paraborealis Tabet, 1979.

Aphoebantus paraborealis Tabet, 2001b: 46. TYPE LOCALITY: USA (Idaho) [H in CAS].

DISTRIBUTION: Nearctic: USA (Idaho).

\section{Genus CONONEDYS Hermann}

\section{bilobata}

Aphoebantus bilobata Bezzi, 1924.

DistRIBUTION: add: Palaearctic: Egypt. inornata

Aphoebantus inornatus Greathead, 1967.

DISTRIBUTION: add: Palaearctic: Jordan.

\section{scutellata}

Anthrax scutellata Meigen, 1835.

Distribution: delete: Palaearctic: Germany.

\section{stenura}

Anthrax stenura Loew, 1871.

DISTRIBUTION: add: Palaearctic: Jordan.

\section{Tribe EXOPROSOPINI Becker}

Genus DIATROPOMMA Bowden

\section{annettae}

Diatropomma annettae Greathead \& Evenhuis, 2001b: 196. TYPE LOCALITY: Tanzania [H in $\left.\mathrm{BMNH}^{*}\right]$.

DISTRIBUTION: Afrotropical: Tanzania. 


\section{asiatica}

Exoprosopa asiatica Zaitzev, 1972.

DISTRIBUTION: add: Palaearctic: Israel.

\section{bowdeni}

Exoprosopa bowdeni Sánchez-Terrón, 1990.

DisTRIBUTION: add: Palaearctic: France (Corsica), Italy (Sardinia), Spain (including Isla Cabrera, Mallorca).

\section{capucina}

Bibio capucina Fabricius, 1781.

Distribution: delete: Palaearctic: Latvia, Moldova.

circeoides

Exoprosopa circeoides Paramonov, 1928.

DISTRIBUTION: add: Palaearctic: Israel.

\section{cleomene}

Exoprosopa cleomene Egger, 1859.

Distribution: add: Palaearctic: Croatia; delete: Palaearctic: Egypt, Moldova.

\section{decrepita}

Anthrax decrepita Wiedemann, 1828.

DISTRIBUTION: add: Palaearctic: Israel.

dispar

Exoprosopa dispar Loew, 1869. COMB. REV. (returned to Exoprosopa from Heteralonia).

Exoprosopa dispar var. interrupta Paramonov, 1928. SYN. N.

Exoprosopa dispar ssp. paramonovi Evenhuis, 1978. SYN. N.

DisTRIBUTION: add: Palaearctic: Cyprus, Greece (Poros), Ukraine.

\section{efflatouni}

Exoprosopa efflatouni Bezzi, 1925.

DISTRIBUTION: add: Palaearctic: Israel.

enigma

Exoprosopa enigma Greathead \& Evenhuis, 2001b: 199. TYPE LOCALITY: Namibia [H in $\left.\mathrm{NMNW}^{*}\right]$.

DISTRIBUTION: Afrotropical: Namibia. 
fasciata

Exoprosopa fasciata Macquart, 1840.

DISTRIBUTION: add: Nearctic: USA (Connecticut).

glossops

Exoprosopa glossops Greathead, 2001a: 143. TYPE LOCALITY: Nigeria [H in $\mathrm{BMNH}^{*}$

DISTRIBUTION: Afrotropical: Nigeria.

jacchus

Anthrax jacchus Fabricius, 1805.

DistRIBUTION: revised: Palaearctic: Albania, Armenia, Austria, Azerbaijan, Bosnia-Herzegovina, Bulgaria, Croatia, France, Gruzia, Hungary, Iran, Italy (including Capri, Sicily), Portugal, Romania, Spain, Yugoslavia.

minois

Exoprosopa minois Loew, 1869.

DISTRIBUTION: add: Palaearctic: Israel.

$\operatorname{minos}$

Anthrax minos Meigen, 1804.

DistRIBUTION: add: Palaearctic: Greece (Ikaria, Kephalonia, Poros, Samos, Seriphos), Jordan; delete: Palaearctic: Czech Republic, Germany.

mira

Exoprosopa mira Hesse, 1936.

DisTRIBUTION: add: Afrotropical: Namibia.

onusta

Anthrax onusta Walker, 1852.

Distribution: add: Palaearctic: Italy (incl. Sicily).

pallasii

Anthrax pallasii Wiedemann, 1818.

DISTRIBUTION: add: Palaearctic: Italy (Sardinia).

pandora

Exoprosopa baccha Loew, 1869 (synonymy in Greathead, 2001c: 280).

DistRIBUTION: revised: Palaearctic: Algeria, Croatia, Greece (including Corfu, Crete, Ithaca, Kephalonia, Lesbos, Rhodes, Zakynthos), Gruzia, Hungary, Israel (including West Bank), Jordan, Libya, Macedonia, Morocco, Tunisia, Turkey, Yugoslavia (Montenegro). 
rivularis

Anthrax rivularis Meigen in Wiedemann, 1818. COMB. N. (from Heteralonia).

DisTRIBUTION: add: Palaearctic: ?Azores Islands, Greece (incl. Kephalonia, Lesbos), Jordan.

rutila

Anthrax rutila Pallas \& Wiedemann in Wiedemann, 1818.

DISTRIBUTION: add: Palaearctic: Italy (Sardinia, Sicily).

selenops

Exoprosopa selenops Greathead, 2001a: 142. TYPE LOCALITY: Kenya [H in BMNH*]. DisTRIBUTION: Afrotropical: Kenya, Tanzania.

\section{Genus HETERALONIA Rondani}

Subgenus ACRODISCA Bezzi

aridicola

Exoprosopa aridicola Hesse, 1956.

DisTRIBUTION: add: Afrotropical: Namibia.

\section{fumida}

Heteralonia fumida Greathead, 2000: 62. TYPE LOCALITY: Namibia [H in NMNW*]

DISTRIBUTION: Afrotropical: Namibia.

\section{obscuripennis}

Exoprosopa obscuripennis Hesse, 1956.

DISTRIBUTION: add: Afrotropical: Namibia.

suffusa

Anthrax suffusa Klug, 1832.

DISTRIBUTION: add: Palaearctic: Jordan.

zonata

Exoprosopa zonata Hesse, 1936.

DisTRIBUTION: add: Afrotropical: Namibia.

Subgenus HOMOLONIA Bowden

aegina

Anthrax aegina Wiedemann, 1828.

DistRIBUTION: add: Palaearctic: Israel. 
Anthrax megerlei Meigen, 1820.

Distribution: add: Palaearctic: France (Corsica), Greece (Crete, Kephalonia, Rhodes), Jordan; delete: Palaearctic: Switzerland.

\section{Subgenus ISOTAMIA Bezzi}

azaniae

Heteralonia azaniae Greathead \& Evenhuis, 2001b: 201. TYPE LOCALITY: Kenya [H in $\mathrm{BMNH}^{*}$ ].

Distribution: Afrotropical: Kenya, Tanzania.

\section{Subgenus ZYGODIPLA Bezzi}

aeaca

Anthrax aeacus Meigen, 1804.

Distribution: add: Palaearctic: Cyprus, Israel; delete: Palaearctic: Switzerland.

algira

Anthrax algira Fabricius, 1794.

Distribution: add: Palaearctic: Croatia.

\section{bagdadensis}

Anthrax bagdadensis Macquart, 1840.

DistRIBUTION: add: Palaearctic: Egypt, Jordan.

bezzii

Exoprosopa bezzii Paramonov, 1928.

DistRIBUTION: add: Palaearctic: Israel.

\section{hermanni}

Exoprosopa hermanni François, 1967.

Distribution: add: Palaearctic: Jordan.

mira

Heteralonia mira Greathead, 2000: 63. TYPE LOCALITY: Namibia [H in NMNW*]

DISTRIBUTION: Afrotropical: Namibia.

\section{mucorea}

Anthrax mucorea Klug, 1832.

DistRIBUTION: add: Palaearctic: Jordan. 
normalis

Exoprosopa normalis Loew, 1869.

DisTRIBUTION: add: Palaearctic: Greece (Crete).

singularis

Exoprosopa singularis Macquart, 1840.

DistRIBUTION: add: Palaearctic: Jordan.

\section{Genus LIGYRA Newman}

satyrus

Bibio satyrus Fabricius, 1775.

DISTRIBUTION: add: Oriental: Singapore.

vittata

Hyperalonia vittata Ricardo, 1901.

DISTRIBUTION: add: Afrotropical: Namibia.

\section{Genus LITORHINA Bowden}

\section{flammea}

Litorhina flammea Greathead \& Evenhuis, 2001b: 205. TyPE LOCALITY: Sierra Leone [H in MZLU*].

DISTRIBUTION: Afrotropical: Sierra Leone.

nyasae

Exoprosopa nyasae Ricardo, 1901.

DISTRIBUTION: add: Afrotropical: Namibia.

vernayi

Litorrhynchus vernayi Hesse, 1956.

DisTRIBUTION: add: Afrotropical: Namibia.

Genus MICOMITRA Bowden

iris

Exoprosopa iris Loew, 1869.

DisTRIBUTION: add: Palaearctic: Jordan. 
zootaxA lapidoti

300 Micomitra lapidoti Zaitzev, 1999c: 717 [2000a: 651]. TYPE LOCALITY: Israel [H in UNKN].

DistRIBUTION: Palaearctic: Israel.

shelkovnikovi

Exoprosopa shelkovnikovi Paramonov, 1928. COMB. N. (transferred to Micomitra from Pterobates).

stupida

Bibio stupida Rossi, 1790.

Distribution: add: Palaearctic: Cyprus, Greece (Corfu), Israel, Macedonia; delete: Palaearctic: Germany.

\section{Genus PTEROBATES Bezzi}

chalybaeus

Exoprosopa chalybea Röder, 1887.

DistrIBUTION: add: Palaearctic: Israel.

\section{Tribe PROROSTOMATINI Hull}

Genus EPACMOIDES Hesse

\section{xerophilus}

Epacmoides xerophilum Hesse, 1956.

Distribution: add: Afrotropical: Namibia.

\section{Genus PLESIOCERA Macquart}

algira

Plesiocera algira Macquart, 1840.

DistRIBUTION: add: Palaearctic: Jordan, Portugal.

philerema

Plesiocera philerema Hesse, 1956.

Distribution: add: Afrotropical: Namibia. 


\section{integrum}

Plesiocera integra Bezzi, 1922.

DisTRIBUTION: add: Afrotropical: Namibia.

\section{Genus STOMYLOMYIA Bigot}

europaea

Tomomyza europaea Loew, 1869.

Distribution: add: Palaearctic: Greece (Crete), Jordan; delete: Palaearctic: Hungary.

tenella

Tomomyza tenella Loew, 1869.

Distribution: add: Palaearctic: Portugal.

Tribe VILLINI Hull

Genus CAECANTHRAX Greathead

arabicus

Anthrax arabica Macquart, 1840.

Distribution: add: Palaearctic: Greece (Crete).

Genus DIPALTA Osten Sacken

serpentina

Anthrax serpentina Osten Sacken, 1877.

DisTRIBUTION: add: Nearctic: USA (North Carolina).

\section{Genus EXHYALANTHRAX Becker}

aberrans

Thyridanthrax aberrans Hesse, 1956.

DisTRIBUTION: add: Afrotropical: Namibia.

afer

Anthrax afer Fabricius, 1794. 
Distribution: add: Palaearctic: Greece (Corfu, Crete, Rhodes), Jordan, Lithuania, Spain (Formentera).

\section{contrarius}

Villa contraria Becker, 1916.

DisTRIBUTION: add: Palaearctic: Greece (Rhodes), Jordan.

\section{finidrontis}

Exhyalanthrax finidrontis Evenhuis \& Greathead, 1999.

DISTRIBUTION: add: Afrotropical: Namibia.

\section{melanchlaenus}

Anthrax melanchlaenus Loew, 1869.

Thyridanthrax albicingulus Austen, 1936. SYN. N.

Thyridanthrax amoenus Austen, 1937. SYN. N.

DistRIBUTION: add: Palaearctic: Greece (Kephalonia), Iran, Jordan, Lebanon.

\section{muscarius}

Anthrax muscaria Pallas in Wiedemann, 1818.

Thyridanthrax innocens Austen, 1936. SYN. N.

DistRIBUTION: add: Palaearctic: Cyprus, Greece (Poros), Israel (incl. Gaza Strip), Jordan, Syria; delete: Palaearctic: Germany.

niveifrons

Thyridanthrax niveifrons Hesse, 1956.

DISTRIBUTION: add: Afrotropical: Namibia.

\section{Genus HEMIPENTHES Loew}

\section{exoprosopoides}

Hemipenthes exoprosopoides Paramonov, 1928.

DISTRIBUTION: add: Palaearctic: Israel.

maura

Musca maura Linnaeus, 1758.

DistRIBUTION: delete: Palaearctic: Netherlands.

morio

Musca morio Linnaeus, 1758.

DistRIBUTION: add: Palaearctic: Andorra, Bosnia-Herzegovina, Germany, Greece (Cephalonia, Crete, Rhodes), Portugal; delete: Palaearctic: Lithuania. 
praecisa

Anthrax praecisus Loew, 1869.

DisTRIBUTION: add: Palaearctic: Israel.

velutina

Anthrax velutina Meigen, 1820.

Distribution: add: Palaearctic: Greece (Kephalonia, Thasos), Jordan.

villeneuvi

Hemipenthes villeneuvi François, 1970.

Distribution: add: Palaearctic: Spain, Switzerland.

\section{Genus OESTRANTHRAX Bezzi}

\section{brunnescens}

Anthrax brunnescens Loew, 1857.

DISTRIBUTION: add: Palaearctic: Jordan.

pallifrons

Oestranthrax pallifrons Bezzi, 1926.

Distribution: add: Palaearctic: Morocco; delete: Palaearctic: Spain.

\section{Genus PACHYANTHRAX François}

\section{fulvifacies}

Thyridanthrax fulvifacies Austen, 1937.

DISTRIBUTION: add: Palaearctic: Jordan.

nimrodicus

Pachyanthrax nimrodica Zaitzev, 1999.

DISTRIBUTION: add: Palaearctic: Jordan.

[Note: Generic status uncertain but it is not Pachyanthrax]

\section{telamon}

Exoprosopa telamon Loew, 1869.

DISTRIBUTION: add: Palaearctic: Greece (Rhodes), Jordan.

unctus

Anthrax unctus Loew, 1869.

DistRIBUTION: add: Palaearctic: Greece (Poros). 


\section{floridensis}

Paravilla floridensis Hall, 1981.

DISTRIBUTION: add: Nearctic: USA (?Connecticut).

\section{Genus THYRIDANTHRAX Osten Sacken}

alphonsi

Thyridanthrax alphonsi Sánchez-Terrón \& Roldán Bravo, 2000: 68. TYPE LOCALITY: Portugal [H in MNCN].

Distribution: Palaearctic: Portugal, Spain.

\section{elegans ssp. elegans}

Anthrax elegans Wiedemann in Meigen, 1820.

Distribution: add: Palaearctic: Greece (Kephalonia, Rhodes), Jordan, Macedonia, Spain (incl. Ibiza, Mallorca).

\section{hispanus}

Anthrax hispanus Loew, 1869. STAT. REV. (removed from synonymy with Thyridanthrax perspicillaris (Loew, 1869)).

DisTRIBUTION: Palaearctic: Spain.

\section{incanus}

Anthrax incana Klug, 1832.

Distribution: add: Palaearctic: Greece (Kephalonia, Poros, Rhodes), Jordan.

lotus

Anthrax lotus Loew, 1869.

Distribution: add: Palaearctic: Greece (Poros), Jordan; delete: Palaearctic: Spain.

mutilus

Anthrax mutilus Loew, 1869.

DistRIBUTION: add: Palaearctic: Greece (Corfu).

nebulosus

Anthrax nebulosus Dufour, 1852.

DistRIBUTION: add: Palaearctic: Portugal.

\section{obliteratus}

Anthrax obliterata Loew, 1862. 
DistRIBUtion: add: Palaearctic: Cyprus, France (Corsica), Greece (Corfu).

perspicillaris ssp. perspicillaris

Anthrax perspicillaris Loew, 1869.

DistRIBUTION: add: Palaearctic: ?Azores, France (Corsica), Greece (Kephalonia), Italy (Sardinia), Jordan, Portugal, Switzerland; delete: Palaearctic: Germany.

polyphemus

Anthrax polyphemus Wiedemann in Meigen, 1820.

DisTRIBUTION: add: Palaearctic: Croatia, Cyprus, Greece (Corfu), Italy (Sicily), Jordan, Macedonia.

Genus VERIBUBO Evenhuis

\section{angusteoculatus}

Anthrax angusteoculatus Becker, 1902.

DisTRIBUTION: add: Palaearctic: Italy (Sicily).

anus

Anthrax anus Wiedemann, 1828.

DISTRIBUTION: add: Palaearctic: Jordan.

misellus

Anthrax misellus Loew, 1869.

DisTRIBUTION: add: Palaearctic: Greece (Poros), Jordan.

saffra

Veribubo saffra Greathead, 1981.

Distribution: add: Palaearctic: Jordan.

\section{Genus VILLA Lioy}

\section{abbadon}

Anthrax abbadon Fabricius, 1794.

DistRIBUTION: add: Palaearctic: France (Corsica), Russia (SET).

\section{bivirgata}

Villa bivirgata Austen 1937.

Distribution: add: Palaearctic: Jordan. 
Villa brunnea Becker, 1916.

DistRIBUTION: add: Palaearctic: Greece (Lesbos, Thasos).

cana

Anthrax cana Meigen, 1804.

Distribution: add: Palaearctic: Cyprus, Italy (Sardinia); delete: Palaearctic:

Czech Republic, Switzerland.

ceballosi

Villa ceballosi Andréu Rubio, 1959.

Distribution: delete: Palaearctic: Spain.

\section{cingulata}

Anthrax cingulata Meigen, 1804.

DisTRIBUTION: add: Palaearctic: Greece (Corfu), ?Norway, Spain (Balearic Islands); delete: Palaearctic: Netherlands.

\section{cingulum}

Anthrax cingulum Wiedemann in Meigen, 1820.

Distribution: delete: Palaearctic: Belgium.

\section{deludens}

Villa deludens François, 1966.

DISTRIBUTION: add: Palaearctic: Croatia.

\section{fasciata}

Anthrax fasciata Meigen, 1804.

Anthrax venusta Meigen, 1820. SYN. N.

Anthrax margaritifer Dufour, 1833. SYN. N.

Anthrax dolosa Jaennicke, 1867. SYN. N.

Anthrax turbidus Loew, 1869. SYN. N.

DistRIBUTION: add: Palaearctic: China, Egypt, France (Corsica), Germany, Iran, Mongolia, Netherlands, ?Norway, Tajikistan.

\section{fasciculata}

Villa fasciculata Becker, 1916.

Villa orientalis Zaitzev, 1966. SYN. N.

Distribution: add: Palaearctic: Armenia, Azerbaijan, Croatia, Greece (Kephalonia, Lefkas, Poros), Gruzia, Jordan, Kazakhstan, Mongolia, Spain (Menorca), Turkmenistan, Uzbekistan. 
halteralis

Anthrax halteralis Kowarz, 1883.

DISTRIBUTION: add: Palaearctic: ?Norway.

\section{hottentotta}

Musca hottentotta Linnaeus, 1758.

Distribution: add: Palaearctic: Andorra, France (Corsica), Greece (Corfu,

Crete, Kephalonia, Thasos), Spain (Isla Cabrera, Mallorca); delete: Palaearctic:

Lithuania, Netherlands, UK (Great Britain and Northern Ireland).

hybrida

Villa hybrida Hesse, 1956.

DISTRIBUTION: add: Afrotropical: Namibia.

insignis

Villa insignis Austen, 1937.

DISTRIBUTION: add: Palaearctic: Jordan.

ixion

Anthrax ixion Fabricius, 1794.

Anthrax humilis Ruthe, 1831. SYN. N.

Anthrax mucida Zeller, 1840. SYN. N.

Anthrax pygarga Loew, 1869. SYN. N.

Anthrax albulus Loew, 1869. SYN. N.

Villa quinta Becker, 1916. SYN. N.

DistRIBUTION: add: Palaearctic: Belgium, Bosnia-Herzegovina, Bulgaria, Egypt,

Greece (Corfu, Crete, Kephalonia, Rhodes), Iran, Jordan, Macedonia, Portugal, Yugoslavia.

\section{leucostoma}

Anthrax leucostoma Meigen, 1820.

Distribution: add: Palaearctic: Greece.

modesta

Anthrax modesta Meigen, 1820.

Distribution: add: Palaearctic: Austria, Netherlands, ?Norway, Portugal, Switzerland; delete: Palaearctic: Poland.

nigrifrons

Anthrax nigrifrons Macquart in Webb \& Berthelot, 1839.

Distribution: add: Palaearctic: Madeira. 
Anthrax niphobletus Loew, 1869.

DistRIBUTION: add: Palaearctic: Greece (Crete, Lesbos), Jordan.

occulta

Anthrax occulta Wiedemann in Meigen, 1820.

Distribution: add: Palaearctic: Sweden, Switzerland; delete: Palaearctic:

Lithuania.

panisca

Bibio panisca Rossi, 1790.

DistRIBUTION: delete: Palaearctic: Belgium, Ireland, Netherlands, Slovakia.

parilis

Villa parilis François, 1969.

Distribution: add: Palaearctic: Slovakia; delete: Palaearctic: Czech Republic.

stenozona

Anthrax stenozona Loew, 1869.

Distribution: add: Palaearctic: Croatia.

Genus VILLOESTRUS Paramonov

uvarovi

Villoestrus uvarovi Paramonov, 1931.

DistRIBUTION: add: Palaearctic: Turkey.

Tribe XERAMOEBINI Hull

Genus DESMATONEURA Williston

nivea

Bibio nivea Rossi, 1790.

DISTRIBUTION: add: Palaearctic: Greece (Crete, Rhodes).

sica

Desmatoneura sica Greathead, 1969.

DisTRIBUTION: add: Afrotropical: Yemen (Abd-el-Kuri). 
albula

Petrorossia albula Zaitzev, 1962.

Distribution: add: Palaearctic: Egypt, Israel, Jordan.

autumnalis

Petrorossia autumnalis Zaitzev, 2002c: 442. TYPE LOCALITY: Kazakhstan [H in ZIS].

Distribution: Palaearctic: Kazakhstan, Kyrgyz Republic.

carmelica

Petrorossia carmelica Zaitzev, 1999c: 703 [2000a: 640]. TYPE LOCALITY: Israel [H in UNKN].

DISTRIBUTION: Palaearctic: Israel.

\section{flavipennis}

Petrorossia flavipennis Zaitzev, 1966.

DisTRIBUTION: add: Palaearctic: Egypt, Israel.

\section{freidbergi}

Petrorossia freidbergi Zaitzev, 1999c: 705 [2000a: 641]. TYPE LOCALITY: Israel [H in UNKN].

DistRIBUTION: Palaearctic: Israel.

\section{fulvipes}

Anthrax fulvipes Loew, 1860.

DISTRIBUTION: add: Afrotropical: Namibia.

\section{guanchorum}

Petrorossia guanchorum François, 1970.

quanchorum, error for guanchorum.

[N.B.: Zaitzev (2000b) records this Canary Island endemic from Israel, which we feel is an extremely unlikely extension of its distribution.]

\section{gussakovskiji}

Petrorossia gussakovskiji Zaitzev, 2000b: 482 [2001b: 209]. TYPE LOCALITY: Tajikistan [H in ZIS].

DISTRIBUTION: Palaearctic: Tajikistan, Turkmenistan, Uzbekistan.

\section{hespera}

Bibio hespera Rossi, 1790.

Petrorossia chraminensis Zaitzev, 1962. SYN. N. 
Petrorossia dobrogica Dusa, 1966. SYN. N.

Distribution: add: Palaearctic: Greece (Kephalonia, Rhodes, Seriphos, Symi, Tylos), Jordan, Spain (Mallorca, Menorca).

\section{iskanderika}

Petrorossia iskanderika Zaitzev, 2000b: 484 [2001a: 210]. TYPE LOCALITY: Tajikistan [H in ZIS].

DisTRIBUTION: Palaearctic: Tajikistan.

\section{israeliensis}

Petrorossia israeliensis Zaitzev, 1999c: 707 [2000a: 643]. TYPE LOCALITY: Israel [H in UNKN].

DISTRIBUTION: Palaearctic: Israel.

letho

Anthrax letho Wiedemann, 1828.

DISTRIBUTION: add: Palaearctic: Jordan.

\section{lucidipennis}

Petrorossia lucidipennis Zaitzev, 1966.

DisTRIBUTION: add: Palaearctic: Israel.

margaritae

Petrorossia margaritae Zaitzev, 1999c: 708 [2000a: 644]. TYPE LOCALITY: Israel [H in UNKN].

DISTRIBUTION: Palaearctic: Israel.

modesta

Petrorossia modesta Zaitzev, 1966.

DISTRIBUTION: add: Palaearctic: Israel.

\section{rufiventris}

Petrorossia rufiventris Zaitzev, 1966.

DisTRIBUTION: add: Palaearctic: Israel.

\section{shibanovae}

Petrorossia shibanovae Zaitzev, 1999c: 710 [2000a: 646]. TYPE LOCALITY: Israel [H in UNKN].

DISTRIBUTION: Palaearctic: Israel.

\section{stackelbergi}

Petrorossia stackelbergi Zaitzev, 2000b: 485 [2001b: 211]. TYPE LOCALITY: Tajikistan [H in ZIS]. 
Distribution: Palaearctic: Tajikistan, Turkmenistan.

\section{volkovitshi}

Petrorossia volkovitshi Zaitzev, 1999c: 712 [2000a: 647]. TYPE LOCALITY: Israel [H in UNKN].

DISTRIBUTION: Palaearctic: Israel.

\section{Genus PIPUNCULOPSIS Bezzi}

arenivaga

Pipunculopsis arenivaga Zaitzev, 2001b: 913 [2001c: 579]. TYPE LOCALITY: Turkmenistan [H in TAU].

[Note: According to the ICZN Code Article 16.4.2, this name was invalid when it was originally proposed in 2001 because it lacked an indication of the type depository. By indication of the type depository here and back reference to the original description, the name is hereby validated and should keep the original authorship of "Zaitzev".]

DISTRIBUTION: Palaearctic: Israel, Kazakhstan, Turkmenistan.

\section{bivittata}

Pipunculopsis bivittata Bezzi, 1925.

DISTRIBUTION: add: Palaearctic: Israel.

\section{deserta}

Pipunculopsis deserta Zaitzev, 2001b: 915 [2001c: 582]. TYPE LOCALITY: Israel [H in TAU].

[Note: According to the ICZN Code Article 16.4.2, this name was invalid when it was originally proposed in 2001 because it lacked an indication of the type depository. By indication of the type depository here and back reference to the original description, the name is hereby validated and should keep the original authorship of "Zaitzev".]

DISTRIBUTION: Palaearctic: Israel, Turkmenistan.

\section{stackelbergi}

Pipunculopsis stackelbergi Zaitzev, 2001b: 917 [2001c: 583]. TYPE LOCALITY: Turkmenistan [H in TAU].

[Note: According to the ICZN Code Article 16.4.2, this name was invalid when it was originally proposed in 2001 because it lacked an indication of the type depository. By indication of the type depository here and back reference to the original description, the name is hereby validated and should keep the original authorship of "Zaitzev".]

DISTRIBUTION: Palaearctic: Israel, Jordan, Kazakhstan, Tajikistan, Turkmenistan. 
XERAMOEBA Hesse, 1956.

Gazlia Zaitzev, 1999b: 458 [2000c: 428]. Type species Gazlia albula Zaitzev, 1999, by original designation. SYN. N

Cazlia: Zaitzev, 1999b: 458 (incorrect original spelling of Gazlia).

albula

Gazlia albula Zaitzev, 1999b: 458 [2000c: 429]. TYPE LOCALITY: Uzbekistan [H in ZIS]. COMB. N.

DISTRIBUTION: Palaearctic: Uzbekistan.

kinereti

Chiasmella kinereti Zaitzev, 1999b: 462 [2000c: 432]. TYPE LOCALITY: Israel [H in TAU]. COMB. $\mathbf{N}$.

DISTRIBUTION: Palaearctic: Israel.

rubicunda

Chiasmella sabulonis var. rubicunda Bezzi, 1925 [L designated by El Hawagry (2001a: 155) in ESEC].

sabulonis

Argyromoeba sabulonis Becker, 1906.

Petrorossia latifrons Bezzi, 1925 [H found! in ESEC] (transferred from Petrorossia to Xeramoeba and synonymy in El Hawagry, 2001: 155).

DISTRIBUTION: add: Palaearctic: Jordan.

salwae

Xeramoeba salwae El Hawagry, 2001: 156. TyPE LOCALITY: Egypt [H in CUE].

DisTRIBUTION: Palaearctic: Egypt, Jordan.

semirufa

Chionamoeba semirufa Sack, 1909.

DISTRIBUTION: add: Palaearctic: Jordan.

stackelbergi

Chiasmella stackelbergi Zaitzev, 1999b: 460 [2000c: 431]. TYPE LOCALITY: Tajikistan [H in ZIS]. COMB. N.

Distribution: Palaearctic: Tajikistan.

turkmenica

Chiasmella turkmenica Zaitzev, 2002c: 440. TYPE LOCALITY: Turkmenistan [H in ZIS]. COMB. N.

Distribution: Palaearctic: Turkmenistan. 
El-Hawagry, M.S. (1999) Two new species of genus Anthrax Scopoli (Bombyliidae-Diptera) from Egypt. Bulletin of the Entomological Society of Egypt, 76[1998], 107-114. (31 December 1999+)

El-Hawagry, M.S. (2001) Revision of the genus Xeramoeba Hesse (Bombyliidae, Diptera) from Egypt, with description of a new species. Studia Dipterologica, 8, 153-159. (15 August 2001)

El Hawagry, M.S. (2002) Three new species of anthracine flies (Diptera: Bombyliidae) from Egypt. Zootaxa, 111, 1-8. (26 November 2002)

El Hawagry, M.S., El Moursy, A.A., Gilbert, F. \& Zalat, S. (2000) The tribe Anthracini Latreille (Bombyliidae, Diptera) from Egypt. Egyptian Journal of Biology, 2, 97-117. (31 December 2000+)

Greathead, D.J. (2000) The family Bombyliidae (Diptera) in Namibia, with descriptions of six new species and an annotated checklist. Cimbebasia, 16, 55-93. (21 March 2000)

Greathead, D.J. (2001a) A study of the Exoprosopa busiris (Jaennicke) group of Bezzi (1924) in tropical Africa (Diptera: Bombyliidae). Journal of Natural History, 35(1), 127-147. (January 2001)

Greathead, D.J. (2001b) Notes on the Geron gibbosus Olivier, 1789 and G. halteralis Wiedemann, 1820 species groups (Diptera: Bombyliidae). II - additional species and records from Europe and Asia. Studia Dipterologica, 8(1), 161-173. (15 August 2001)

Greathead, D.J. (2001c) Exoprosopa pandora (Fabricius, 1805) (Diptera: Bombyliidae) and related species in Europe and the Mediterranean Basin. Insect Systematics and Evolution, 32, 279-284. (October 2001)

Greathead, D.J. (2003) Notes on Anthrax dentata (Becker, 1906), A. trifasciatus Meigen, 1804, and related species of Bombyliidae (Diptera) in Africa and Eurasia. Deutsche Entomologische Zeitschrift, 50(1), 89-94. (30 June 2003)

Greathead, D.J. \& Evenhuis, N.L. (2001a) Bombylioidea (Diptera: Bombyliidae; Mythicomyiidae) from the island of Sokotra. Zootaxa 14, 11 pp. (26 November 2001)

Greathead, D.J. \& Evenhuis, N.L. (2001b) Annotated keys to the Bombylioidea (Diptera: Bombyliidae; Mythicomyiidae) of Africa. African Invertebrates, 47, 105-244. (15 December 2001)

Hall, J.C. \& Evenhuis, N.L. (2003) Review of the genus Geron (Geron) Meigen in the Nearctic Region (Diptera: Bombyliidae: Toxophorinae). Zootaxa, 181, 72 pp. (16 April 2003)

Hasbenli, A. \& Zaitzev, V.F. (2000) Two new species of Bombylius Linnaeus, 1758 (Diptera, Bombyliidae) from Turkey. Deutsche Entomologisches Zeitschrift, 47, 105-118. (26 June 2000)

Lamas, C.J.E. \& Couri, M.S. (1999) Revisião de Euprepina Hull (Diptera, Bombyliidae, Bombyliinae). Revista Brasileira de Zoología, 16(2), 461-482. (June 1999)

Lamas, C.J.E., Evenhuis, N.L. \& Couri, M.S. (2001) Review of the southern African genus Apatomyza Wiedemann (Diptera, Bombyliidae), with descriptions of new species. Studia Dipterologica, 8(1), 175-186. (15 August 2001)

Lamas, C.J.E., Evenhuis, N.L. \& Couri, M.S. (2003) New species and identifications keys of southern African Crocidiinae (Diptera, Bombyliidae). Deutsche Entomologisches Zeitschrift, 50(1), 95-110. (30 June 2003)

Nagatomi, A., Tamaki, N. \& Evenhuis, N.L. (2000) A new Systropus from Taiwan and Japan (Diptera, Bombyliidae). South Pacific Study, 21(1), 15-18. (31 October 2000)

Sánchez-Terrón, A. \& Roldán Bravo, A.V. (2000) El género Thyridanthrax Osten Sacken, 1886 en el área ibero-balear, con la descripción de una nueva especie (Diptera, Bombyliidae). Boletín de la Asociación Española de Entomología, 24(1-2), 65-84. (20 June 2000)

Tabet, A.B. [2001a]. A new species of Toxophora from Idaho (Bombyliidae: Diptera). Journal of Basic and Applied Sciences (Libya), 6[1998], 44. (15 June 2001) 
Tabet, A.B. [2001b]. New North American Bombyliidae (Diptera). Journal of Basic and Applied Sciences (Libya), 6[1998], 45-47. (15 June 2001)

[See Tabet (2001a) annotation for details on dating.]

Tabet, A.B. [2001c]. Three new species of Geron from Idaho (Diptera: Bombyliidae). Journal of Basic and Applied Sciences (Libya), 6[1998], 48-52. (15 June 2001)

[See Tabet (2001a) annotation for details on dating.]

Tabet, A.B. [2001d]. Description of new species of North American beeflies (Diptera: Bombyliidae. Journal of Basic and Applied Sciences (Libya), 6[1998], 53-57. (15 June 2001)

[See Tabet (2001a) annotation for details on dating.]

Zaitzev, V.F. (1999b) A new genus and new species of flies of the family Bombyliidae (Diptera) from the Palaearctic. Entomologicheskoe Obozrenie, 78(2), 457-463. (22 September 1999)

[Date of receipt at ZIS. In Russian; English translation, 2000, in Entomol. Rev. 79(4): 428-433.]

Zaitzev, V.F. (1999c) On the fauna of flies of the family Bombyliidae (Diptera) of Israel. V. Entomologicheskoe Obozrenie, 78(3), 703-718. (31 December 1999)

[Date received by the Russian Entomological Society. In Russian; English translation, 2000a, in Entomol. Rev. 79(6): 640-653.]

Zaitzev, V.F. (2000b) New species of the genus Petrorossia Bezzi (Diptera, Bombyliidae) from Middle Asia. Entomologicheskoe Obozrenie, 79(2), 482-486. (8 August 2000)

[Date of receipt at ZIS. In Russian; English translation, 2001b, in Entomol. Rev. 80(2): 209-212.]

Zaitzev, V.F. [2001a]. Revision of the genus Pipunculopsis Bezzi, 1925 (Diptera, Bombyliidae). Entomologicheskoe Obozrenie, 79(4)[2000], 911-919. (24 January 2001)

[Date of receipt at ZIS. In Russian; English translation 2001c, in Entomol. Rev. 80(5): 578-585.]

Zaitzev, V.F. [2002a]. Three new species of the family Bombyliidae (Diptera from Israel. Entomologicheskoe Obozrenie, 80(4), 896-900. (1 February 2002)

[Date of receipt at ZIS. In Russian; English translation, 2002b, in Entomol. Rev. 81(4): 426-429.]

Zaitzev, V.F. (2002a) New species of the family Bombyliidae (Diptera) from Palaearctic. Entomologicheskoe Obozrenie, 81(2), 439-444. (11 September 2002)

[Date of receipt at ZIS.] 


\section{List of publications treating $U$ sia as a valid genus-group name}

Andreú Rubio, J.M. (1959) Bombílidos marroquíes del Instituto Español de Entomología (Diptera). Eos, 35, 7-19.

Báez, M. (1982) Dos nuevas especies del genero Usia en las Islas Canarias (Diptera, Bombyliidae). Redia, 65, 253-58.

Báez, M. \& Garcia, A. (2001) Order Diptera.. In: Izquierdo, I., et al., Lista de Especies Silvestres de Canarias (Hongos, Plantas y Animales Terrestres) 2001. Consejería de Política Terrioriál y Medio Ambiente Gobierno de Canarias, Tenerife, pp. 249-267.

Bowden, J. (1975) Family Bombyliidae. In: Delfinado, M.D. \& D.E. Hardy, eds., A catalog of the Diptera of the Oriental Region. Vol. II. Suborder Brachycera through Division Aschiza, Suborder Cyclorrhapha. University Press of Hawaii, Honolulu, p. 165-84.

Bowden, J. (1980) Family Bombyliidae. Pp. 381-430. In: Crosskey, R.W. (ed.), A catalogue of the Diptera of the Afrotropical Region. British Museum (Natural History), London, 1,437 pp.

Carles-Tolra, M. (2002) Catalogo de los Diptera de España, Portugal y Andorra (Insecta). Monografías de la Sociedad de Entomológica Aragonesa, 8, 1-319.

Contini, C., Lyneborg, L., Majer, J.M., Rivosecci, L. \& Zaitzev, V.F. (1995) Diptera Nemestrinoidea, Asiloidea, Bombyloidea. Checklist delle specie della fauna Italiana, 68, 1-19.

DuMerle, P. (1971) Biologie de deux espècies de genre Usia Latreille (Diptera: Bombyliidae). Annales de la Société Entomologique de France (n.s.), 7, 241-259.

Ebejer, M. (1988) Beeflies (Dipt., Bombyliidae) from Malta. Entomologist's. Monthly Magazine, 124, 233-241.

Evenhuis, N.L. (1990) Systematics and evolution of the genera of the subfamilies Usiinae and Phthiriinae of the world (Diptera: Bombyliidae). Entomonograph, 11[1989], 1-72.

Evenhuis, N.L. (1991) World catalog of genus-group names of bee flies (Diptera: Bombyliidae). Bishop Museum Bulletin in Entomology, 5, vii + 105 pp.

Evenhuis, N.L. (1994) Catalogue of the Fossil Flies of the World (Insecta: Diptera). Backhuys Publishers, Leiden, $600 \mathrm{pp}$.

Evenhuis, N.L. \& Greathead, D.J. (1999) World Catalog of Bee Flies (Diptera: Bombyliidae). Backhuys Publishers, Leiden, 756 pp.

François, F.J. (1969) Bombyliidae (Diptera) from southern Spain, with descriptions of twelve new species. Entomologisk Meddelelser, 37, 107-60.

Greathead, D.J. (1988) Diptera: Fam. Bombyliidae of Saudi Arabia (part 2). In: Büttiker, W. \& F. Krupp, (eds.), Fauna of Saudi Arabia. Vol. 9. Pro Entomologica, Basle, Switzerland, pp. 90113.

Greathead, D.J. \& Evenhuis, N.L. (1997) Family Bombyliidae. In: Papp, L. \& Darvas, B. (eds.), Contributions to a Manual of Palaearctic Diptera (with Special Reference to Flies of Economic Importance). Volume 2. Nematocera to lower Brachycera. Science Herald, Budapest, pp. 487512.

Greathead, D.J. \& Evenhuis, N.L. (2001) Annotated keys to the genera of African Bombylioidea (Diptera: Bombyliidae; Mythicomyiidae). African Invertebrates, 42, 105-224.

Hall, J.C. [1976]. The Bombyliidae of Chile. University of California Publications in Entomology, 76[1975], 1-278.

Mühlenberg, M. (1971) Phylogenetisch-systematische Studien an Bombyliiden (Diptera). Zeitschrift für Morphologie und Ökologie der Tiere, 70, 73-102.

Muhlenberg, M. (1974) Das Paarungsverhalten von Ceratolaemus Hesse sp. (Diptera, Bombyliidae, Cyrtosiinae) im Vergleich zu dem anderer Wollschweber. Zeitschrift für Tierpsychologie, 33, 437-460. 
Sphon, G.G. (1973). Additional type specimens of fossil Invertebrata in the collections of the Natural History Museum of Los Angeles County. Los Angeles County Museum Contributions in Science, 250, 1-75.

Yang C.-k. \& Du J.-p. (1994) Two new species of Usiinae (Diptera: Bombyliidae) from China. Entomotaxonomica, 16, 272-274.

Yeates, D.K. (1994) The cladistics and classification of the Bombyliidae (Diptera: Asiloidea). Bulletin of the American Museum of Natural History, 219, $191 \mathrm{pp}$.

Zaitzev, V.F. (1966) [Parasitic Flies of the Family Bombyliidae (Diptera) in the Fauna of Transcaucasia]. Nauka, Moscow \& Leningrad, 375 pp.

Zaitzev, V.F. (1989) Family Bombyliidae. In: Soós, Á. \& L. Papp, L., (eds.), Catalogue of Palaearctic Diptera. Vol. 6. Therevidae-Empididae. Akadémiai Kiadó, Budapest, pp. 43-169.

Zaitzev, V.F. \& Kandybina, M.K. (1983) [Fam. Bombyliidae]. In: Zaitzev, V.F., Kandybina, M.N., Nartshuk, E.P. \& V.A. Rikhter, [Catalog of the type specimens in the collection of the Zoological Institute of the Academy of Sciences of the USSR. Insecta (Diptera). Part 1. Families Acroceridae, Nemestrinidae, Bombyliidae]. "Nauka”, Leningrad [= St. Petersburg], pp. 12-44. 